\title{
MAGNETIC AND GRAVITATIONAL DISK-STAR INTERACTIONS: AN INTERDEPENDENCE OF PMS STELLAR ROTATION RATES AND SPIN-ORBIT MISALIGNMENTS
}

\author{
Konstantin Batygin ${ }^{1}$ and Fred C. Adams ${ }^{2,3}$ \\ ${ }^{1}$ Institute for Theory and Computation, Harvard-Smithsonian Center for Astrophysics, 60 Garden St., Cambridge, \\ MA 02138, USA; kbatygin@cfa.harvard.edu \\ ${ }^{2}$ Department of Physics, University of Michigan, Ann Arbor, MI 48109, USA; fca@umich.edu \\ ${ }^{3}$ Department of Astronomy, University of Michigan, Ann Arbor, MI 48109, USA \\ Received 2013 May 30; accepted 2013 October 6; published 2013 November 13
}

\begin{abstract}
The presence of giant gaseous planets that reside in close proximity to their host stars, i.e., hot Jupiters, may be a consequence of large-scale radial migration through the protoplanetary nebulae. Within the framework of this picture, significant orbital obliquities characteristic of a substantial fraction of such planets can be attributed to external torques that perturb the natal disks out of alignment with the spin axes of their host stars. Therefore, the acquisition of orbital obliquity likely exhibits sensitive dependence on the physics of disk-star interactions. Here, we analyze the primordial excitation of spin-orbit misalignment of Sun-like stars in light of disk-star angular momentum transfer. We begin by calculating the stellar pre-main-sequence rotational evolution, accounting for spin-up due to gravitational contraction and accretion as well as spin-down due to magnetic star-disk coupling. We devote particular attention to angular momentum transfer by accretion, and show that while generally subdominant to gravitational contraction, this process is largely controlled by the morphology of the stellar magnetic field (that is, specific angular momentum accreted by stars with octupole-dominated surface fields is smaller than that accreted by dipole-dominated stars by an order of magnitude). Subsequently, we examine the secular spin-axis dynamics of diskbearing stars, accounting for the time-evolution of stellar and disk properties, and demonstrate that misalignments are preferentially excited in systems where stellar rotation is not overwhelmingly rapid. Moreover, we show that the excitation of spin-orbit misalignment occurs impulsively through an encounter with a resonance between the stellar precession frequency and the disk-torquing frequency. Cumulatively, the model developed herein opens up a previously unexplored avenue toward understanding star-disk evolution and its consequences in a unified manner.
\end{abstract}

Key words: accretion, accretion disks - planets and satellites: formation - protoplanetary disks stars: formation - stars: pre-main sequence

Online-only material: color figures

\section{INTRODUCTION}

The birth and early evolution of planetary systems is a phenomenologically rich physical process. Among the numerous factors that affect this process is the physical evolution of pre-main-sequence (PMS) stars, around which planets form. Consequently, understanding the time-dependence of PMS stellar parameters may be crucial to understanding the primordial shaping of planetary systems.

The appreciation for the role that young stars play in sculpting planetary systems was already recognized in early investigations of solar system formation (see, for example, Sekiya et al. 1980). However, this connection has been strengthened immensely by the discovery and subsequent characterization of extrasolar planets (Wolszczan \& Frail 1992; Mayor \& Queloz 1995; Marcy \& Butler 1996; Charbonneau et al. 2000). Indeed, such early results as the stellar metallicity-planet occurrence correlation (Fischer \& Valenti 2005) have had a tremendous impact on our understanding of planet formation (e.g., Laughlin et al. 2004), while from a historical point of view, it is interesting to note that the first proposed search (via the radial velocity and transit techniques) for inflated giant planets that reside in close proximity to their host stars (now known as hot Jupiters) was inspired by observations of eclipsing binary stars (Struve 1952).

Over the last decade or so, both stellar and planetary astronomy have made considerable observational advances, allowing the scientific frontiers to shift forward. With a relatively well-established understanding of the main-sequence evolution of Sun-like stars, stellar-oriented observational surveys have began to focus on elucidating the distribution of rotation rates (Herbst et al. 2007) as well as magnetic field properties (Gregory et al. 2012) of PMS T Tauri stars. Over the same time period, the aggregate of transiting hot Jupiters has grown to an appreciable size, and observations of the Rossiter-McLaughlin effect (Rossiter 1924; McLaughlin 1924) have revealed that the orbits of some hot Jupiters are significantly misaligned with respect to the spin axes of their host stars (Winn et al. 2007, 2011a, 2011b).

A plausible explanation for the close-in orbits of hot Jupiters is orbital migration, driven by viscous evolution of the protoplanetary disks ${ }^{4}$ (Goldreich \& Tremaine 1980; Lin et al. 1996). Within the context of this model, planets arrive to their shortperiod (of the order of $\sim 3$ days) orbits early on, allowing disk-star coupling to partially dictate the range of viable orbital obliquities. Accordingly, the primary purpose of this work is to consider protoplanetary disk-star interactions and propose a framework in which spin-orbit misalignments and PMS stellar evolution fit into a unified picture. Before engaging in calculation, let us first briefly review some of the relevant observational studies.

By the turn of this century, thousands of PMS stellar rotation rate measurements had been performed (Herbst et al. 2002).

\footnotetext{
4 The same explanation is often invoked to account for the assembly of giant planets into mean motion resonances (Lee \& Peale 2002; Morbidelli \& Crida 2007).
} 
This increase in available data has provided estimates for the width of the PMS spin period distribution. Specifically, in the observational sample of Littlefair et al. (2010), the rotation periods are distributed such that most stars lie in the range of $P=1-10$ days (although some stars rotate as quickly as $P=0.6$ days and some as slowly as $P=20$ days). In addition, this sample shows a bimodality in the period distribution, with the two peaks centered around $P=2$ and $P=8$ days (Herbst et al. 2007). However, these studies use ground-based observations that could be subject to uncertainty.

A competing set of space-based observations, the COROT survey of NGC 2264 (Affer et al. 2013), has a longer time baseline and does not find a significant bimodal distribution (see their Figure 5). Nonetheless, the width of the distribution is nearly the same, with most periods falling in the range of $P=1-10$ days (with extrema at $\sim 0.5$ and $\sim 19$ days). Moreover, there appears to be evidence for disk-star coupling in the observational sample, as weak line T Tauri stars (those without disks) are found to rotate somewhat faster, with a median period of 4.2 days, compared to a median of 7.0 days for classical $\mathrm{T}$ Tauri stars. Furthermore, observations indicate that rotational evolution depends strongly on stellar mass (see Bouvier 2013 for a recent review). For example, rotation periods for lower mass stars $M_{\star} \lesssim 0.4 M_{\odot}$ are shorter than those of higher mass stars by about a factor of $\sim 2$ on average (Lamm et al. 2005).

To explain the observations theoretically, a number of mechanisms that affect angular momentum evolution of the stars have been explored. These include disk-locking, where stellar magnetic fields couple to a nearby sector of the disk and enforce quasi-corotation. Although uncertainties persist (Bouvier 2013), it is possible that this process can naturally slow down the stellar rotation from breakup velocities to periods in the range of $P=1-15$ days (Königl 1991; Shu et al. 1994). Additional angular momentum can be carried away by stellar wind, which acts to further brake the rotation rate (Kawaler 1988; Matt \& Pudritz 2008). Simultaneously, gravitational contraction that takes place along the PMS track acts to spin up the stars (e.g., Donati et al. 2012), as does accretion of disk material (Armitage \& Clarke 1996). It is noteworthy that the aforementioned processes, with the exception of gravitational contraction, inherently depend on the detailed structure of the stellar magnetic field.

In a parallel set of developments, observations of the Zeeman effect (Johns-Krull 2007; Donati et al. 2010) have revealed preferentially dipole and octupole-dominated surface magnetic fields. Importantly, observations suggest a mass and age dependence of the field morphology. As stellar age and mass increase, the overall dipole field decreases slightly while the relative strength of the octupole increases, reaching values as much as an order of magnitude larger than those of the dipole component (Gregory et al. 2012). Qualitatively speaking, this dependence may be expected. More massive stars develop radiative interiors early in their PMS evolution (Hansen \& Kawaler 1994; Phillips 1994), effectively pushing the primary field-generating region to the outer layers of the stars. This development in turn forces the higher-order harmonics of the field to become more pronounced at the surface. It is interesting to note that in some similarity to this picture, the non-dipolar dominated field of Neptune is thought to be a consequence of a stably stratified layer in the planetary interior (Stanley \& Bloxham 2004).

Finally, let us remark on spin-orbit misalignments of transiting planets. Although to date the total number of measurements continues to be relatively small, it is believed that about a fourth of the Hot Jupiter sample exhibits large orbital obliquities
(Wright et al. 2011). Orbital obliquities are typically interpreted as relics associated with the physical mechanism by which giant planets that form beyond the ice-line (Pollack et al. 1996) migrate to short-period orbits. However, Rogers et al. (2012) argue that the observations can be understood as a manifestation of the modulation of stellar surface rotation by internal gravity waves. Even if the conventional interpretation is correct (an assumption we shall make here), the origin of these obliquities remains elusive, as the process by which giant planets arrive onto close-in orbits also remains controversial. Traditionally, hot Jupiters with high orbital obliquities have been attributed to "violent" migration scenarios where an initially distant giant planet gets tidally captured onto a close-in orbit after attaining a nearly parabolic orbit through processes such as planet-planet scattering (Ford \& Rasio 2008; Nagasawa et al. 2008; Beaugé \& Nesvorný 2012), Kozai resonance (Wu \& Murray 2001; Fabrycky \& Tremaine 2007; Naoz et al. 2011), or slow chaotic diffusion (Wu \& Lithwick 2011). Meanwhile "calm" disk-driven (i.e., Type-II) migration (Goldreich \& Tremaine 1980; Lin et al. 1996) was thought to produce spin-orbit aligned planets. However, it has been recently shown that spin-orbit misalignments naturally arise within the framework of disk-driven migration as a result of external gravitational torques exerted on the disks by primordial stellar companions (Batygin 2012). Indeed, such external perturbations are likely if the majority of stars of interest (i.e., solar-type stars) are born in binary or multiple systems (Ghez et al. 1993; Kraus et al. 2011; Marks \& Kroupa 2012). We also note that most stars are born in embedded cluster environments (Lada \& Lada 2003) and other stellar members can also provide external torques via stochastic interactions (Adams 2010; Bate et al. 2010).

Although the disk-torquing model proposed by Batygin (2012) naturally avoids the necessity for specifically molded initial conditions inherent to violent migration models, it is sensitive to the physics of disk-star angular momentum transfer. Intuitively, it can be understood that if accretion of disk material is sufficiently rapid, then the star will continuously realign with the disk and thereby prevent the excitation of significant misalignments (Lai et al. 2011). On the other hand, if the star rotates rapidly enough for its gravitational quadrupole to couple adiabatically to the disk (Henrard 1982), its spin pole will trail the angular momentum vector of the disk. This means that the distributions of stellar rotation rates and spin-orbit misalignments may be intimately connected. In this paper, we propose that both the existence of large spin-orbit misalignments as well as perfect alignments may be understood within the framework of the disk-torquing theory in conjunction with the evolution of stellar spin and disk-star coupling during the PMS stage of the system's lifetime.

It is often the case in astrophysical problems that the input parameters required for the calculation possess significant uncertainties and/or the number of physical processes that need to be treated simultaneously is large enough to limit the precision with which any one phenomenon can be described. In this regard, the model we aim to construct is no exception: we are simultaneously concerned with magnetic torquing of the star by the disk, magnetically controlled accretion of disk material, the structure and physical evolution of the star and the disk, as well as gravitational perturbations of a binary companion on the system. Indeed, each of these processes individually constitutes an active research field. Accordingly, rather than trying to capture as much detail as possible with the aid of numerical simulations, here we concentrate on developing an 


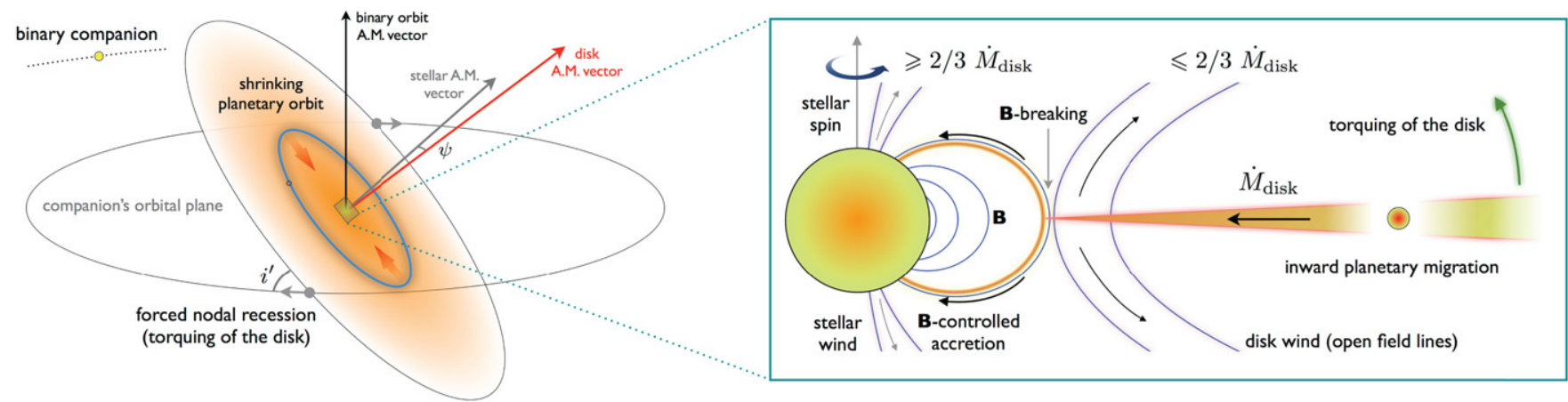

Figure 1. Geometrical setup of the problem. The left side of the figure depicts a protoplanetary disk residing in a binary system, where the disk-binary orbit inclination is $i^{\prime}$. The disk is taken to possess a giant planet, whose orbit slowly shrinks due to the viscous evolution of the gas (type-II migration). The angular momentum vectors of the stellar rotation, binary orbit, and the disk are labeled. Note that the (unprojected) misalignment angle $\psi$ is the angle between the angular momentum vectors of the stellar rotation and the disk. The right side of figure depicts a close-up of the disk-star interface. The blue lines depict the stellar magnetic field, and the accretion of disk material is taken to occur along a critical field line, highlighted with orange on the figure. For completeness, the figure also shows open magnetic field lines associated with the stellar and disk winds. Although disk winds remove a comparatively small fraction of the disk mass (an effect we ignore in the paper), they can carry away a significant fraction of the orbital angular momentum.

(A color version of this figure is available in the online journal.)

intuitive analytical theory, which we then use to shed light on the behavior of the system in the parameter regimes of interest.

This paper is organized as follows. In Section 2, we outline the basic ingredients needed for our calculation, namely, the stellar and disk structure/evolution models. In Section 3, we consider the rotational evolution of young stars. In particular, we consider gravitational contraction, magnetic disk-star torques, as well as accretion. Specific care is taken in treating the magnetically controlled accretion of disk material onto stars, as we show that (contrary to some previous claims) this process is generally sub-dominant to gravitational contraction. Moreover, geometrical considerations imply that objects with octupole-dominated surface fields accrete an order of magnitude less angular momentum than their dipole-dominated counterparts. In Section 4, we study the dynamics of stellar spin axes subject to interactions with a continuously torqued disk, accounting for the evolution of the stellar rotation rate. Our calculations demonstrate that while significant misalignments cannot be excited for rapidly rotating stars, arbitrarily large misalignments can be excited, via resonant encounters, for stars that are successfully spun down. We discuss our results and their implications in Section 5.

\section{MODELS FOR DISK AND STAR EVOLUTION}

The general setup of the model we consider is depicted as a cartoon in Figure 1. As mentioned above, we shall begin by treating the evolution of the stellar spin, accounting for star-disk angular momentum transfer. To complete the specification of the problem, we need to determine the basic parameters for the star and disk. First, we need to specify the mass accretion rate $\dot{M}_{\text {disk }}$ and the disk mass $M_{\text {disk }}$ as functions of time. In the simplest case of isolated disks, possessing no additional sources or sinks of disk material, the two quantities are related via $d M_{\text {disk }} / d t=\dot{M}_{\text {disk }}=-\dot{M}_{\star}$. Here, we adopt this simplifying assumption. For completeness, however, we note that the disk can lose mass through photoevaporation, in addition to accreting mass onto the star. Furthermore, in the embedded phase of evolution, the disk can gain mass from the infalling envelope. Thus, in a more sophisticated iteration of our model, the two quantities $\dot{M}_{\text {disk }}$ and $M_{\text {disk }}$ could be specified independently.

For the sake of definiteness, we use a simple evolutionary model where the disk mass varies with time according to the function

$$
M_{\text {disk }}=\frac{M_{\text {disk }}^{0}}{1+t / \tau}
$$

The corresponding mass accretion rate is then given by

$$
\dot{M}_{\mathrm{disk}}=-\frac{M_{\mathrm{disk}}^{0}}{\tau(1+t / \tau)^{2}},
$$

where we adopt a sign convention so that the accretion rate onto the star is positive. ${ }^{5}$ This general form produces disk masses and disk accretion rates that are roughly consistent with observations (e.g., Hartmann et al. 1998, 2006; Hartmann 2008; Herczeg \& Hillenbrand 2008; Hillenbrand 2008). A reasonable fit to the observed correlations can be obtained using a starting disk mass $M_{\text {disk }}^{0}=0.05 M_{\star}$ and a timescale $\tau_{0}=0.5 \mathrm{Myr}$. This form can also reproduce the observed correlation between the accretion rate and stellar mass, $\dot{M}_{\text {disk }} \propto M_{\star}^{2}$ (Hillenbrand et al. 1992), if we allow the timescale to vary according to the relation $\tau=\tau_{0}\left(M_{\star} / 1 M_{\odot}\right)^{-1}$. However, we note that in practice the accretion of disk material onto young stellar objects is expected to be episodic (e.g., see Caratti o Garatti et al. 2011 and references therein), so the above formulae are intended as approximate, time-averaged representations.

To specify the stellar properties, we utilize traditional polytropic models (Chandrasekhar 1939). After solving the Lane-Emden equation to find the density profile, we determine the dimensionless moment of inertia $I$ and the apsidal motion constant $k_{2}$ as a function of the polytropic index $n$. Both of these quantities are required for the calculation of stellar spin-up, as well as the spin-axis dynamics, and are shown as functions of the polytropic index in Figure 2. Since Sun-like stars (which are the primary focus of this work) are expected to remain (partially) convective throughout much of their disk-bearing lifetime, we can use a polytropic index $n=3 / 2$ to model the interior structure. We note that stars that retain their disks substantially longer than $\sim 4$ Myr will develop significant radiative cores, rendering $n=3$ a more appropriate polytropic index at these latter epochs. Here, we shall ignore this complication, but for reference, both polytropic indexes are labeled in Figure 2.

\footnotetext{
5 Note that in this treatment, the increase in stellar mass associated with accretion is neglected.
} 


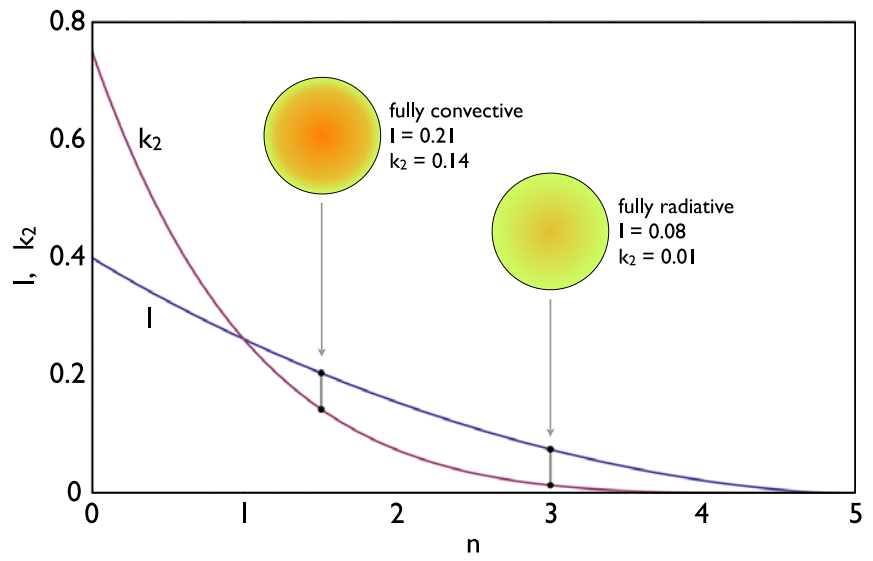

Figure 2. Dimensionless moment of inertia, $I$, and the apsidal motion constant, $k_{2}$, as functions of the polytropic index, $n$. The $n=3 / 2$ polytrope, corresponding to a fully convective star and used throughout the paper, as well as the $n=3$ polytrope, corresponding to a fully radiative star (closer to the present Sun) are labeled.

(A color version of this figure is available in the online journal.)

It is implicitly understood that the radii (and more generally the interior structures) of stars can evolve considerably on the PMS track. In an approximate sense, this evolution can be understood by noting that for a polytrope of index $n$, the energy can be written in the form

$$
E=-\frac{3}{10-2 n} \frac{\mathcal{G} M_{\star}^{2}}{R_{\star}}=-b \frac{\mathcal{G} M_{\star}^{2}}{R_{\star}},
$$

where the second equality defines the dimensionless parameter $b$. To leading order, we can assume that the energy is radiated away according to

$$
\frac{d E}{d t}=-L_{\star}=-4 \pi R_{\star}^{2} \sigma T_{\mathrm{eff}}^{4}=b \frac{\mathcal{G} M_{\star}^{2}}{R_{\star}^{2}} \frac{d R_{\star}}{d t},
$$

where the temperature is kept constant (this assumption is strictly true only as long as the star remains convective). Neglecting changes in the interior distribution of mass (that is, keeping $b=$ constant), this expression can be considered as a differential equation for the stellar radius and has the solution

$$
R_{\star}=\left[\frac{b \mathcal{G} M_{\star}^{2}}{b G M_{\star}^{2} /\left(R_{\star}^{0}\right)^{3}+12 \pi \sigma T_{\mathrm{eff}}^{4} t}\right]^{1 / 3},
$$

where $R_{\star}^{0}$ is the stellar radius at some (arbitrarily chosen) initial condition $t=0$.

\section{EVOLUTION OF STELLAR ROTATION RATES}

With stellar and disk structure models in place, this section considers the evolution of the stellar spin rate due to the physical changes in the stellar radius and interactions with the disk. The primary aim of the following calculations is to identify the dominant effects and establish the conditions under which the observed period distribution can be (even approximately) reproduced. As such, we shall treat each effect in isolation and opt to perform numerical evolutionary calculations only at the end.

Although we only seek to describe stellar rotational evolution during the PMS stage, we must further limit the scope of our treatment to obtain a sound qualitative understanding.
Specifically, here we shall focus on evolution that takes place after the embedded phase of the star-disk system. In terms of absolute stellar age, the embedded phase lasts $\sim 0.1-0.5 \mathrm{Myr}$ on average (Evans et al. 2009). As such, here we shall take the fiducial $t=0$ starting condition as the time when the properties of the young star-disk system first become optically visible. We begin by considering the most physically straightforward process, which is gravitational contraction.

\subsection{Gravitational Contraction}

As already alluded to above, stellar radii undergo drastic changes in the first few Myr of their lives. At birth, the radius of a Sun-like star can exceed that of the present Sun by as much as a factor of $\sim 5$, although by the time such an object emerges from the embedded phase, its radius is down to $R_{\star}^{0} \simeq 4 R_{\odot}$ (Siess et al. 2000). The subsequent contraction of a solar-mass star is well approximated by Equation (5) with $T_{\text {eff }}=4000 \mathrm{~K}$.

In isolation, gravitational contraction conserves angular momentum. The stellar spin-up associated with the reduction of the moment of inertia is governed by the equation

$$
2 I M_{\star} R_{\star} \omega_{\star} \frac{d R_{\star}}{d t}+I M_{\star} R_{\star}^{2} \frac{d \omega_{\star}}{d t}=0 .
$$

Upon substituting Equation (4) into this expression, it is apparent that the spin-up timescale associated with gravitational contraction is

$$
\tau_{R_{\star}}=\frac{b \mathcal{G} M_{\star}^{2}}{8 \pi \sigma T_{\mathrm{eff}}^{4}\left(R_{\star}^{0}\right)^{3}} \sim 1 \mathrm{Myr},
$$

which is essentially the Kelvin-Helmholtz time. This timescale varies substantially with stellar mass and other properties. For instance, $\mathbf{M}$ dwarfs are characterized by considerably larger values of $\tau_{R_{\star}}$ than Sun-like stars (see Stahler \& Palla 2005 for further discussion).

Augmented with Equation (5), Equation (6) admits the solution

$$
\omega_{\star}=\omega_{\star}^{0}\left(1+\frac{12 \pi\left(R_{\star}^{0}\right)^{3} \sigma T_{\mathrm{eff}}^{4}}{b G M_{\star}^{2}} t\right)^{2 / 3}
$$

where $\omega_{\star}^{0}$ is the initial condition. Substituting the stellar parameters adopted above, as well as a typical disk lifetime of $\tau_{\text {disk }} \sim 3$ Myr (Williams \& Cieza 2011) into the above expression, we obtain a factor of $\sim 4$ spin-up during the disk-bearing phase of the star. As will be shown below, the increase in the rotation rate associated with gravitational contraction dominates over that which is associated with the accretion of disk material.

\subsection{Magnetically Controlled Accretion}

In orbit around an unmagnetized object, an accretion disk can be envisioned to extend all the way down to the object's surface. ${ }^{6}$ This picture no longer holds if the central star possesses a magnetic field whose pressure can overcome the ram pressure of the accreting material (Ghosh \& Lamb 1978). Indeed, the $\sim \mathrm{kG}$ fields generally inferred for T Tauri stars are almost certainly strong enough to carve out a substantial cavity within the inner disk (Shu et al. 1994).

\footnotetext{
6 In this case, angular momentum transport is accomplished primarily by (magneto) sonic waves excited in the boundary layer (Narayan et al. 1987; Belyaev et al. 2013).
} 
The truncation radius of the disk is given by the expression

$$
a_{\text {in }}=\bar{c}\left(\frac{8 \pi^{2}}{\mu_{0}^{2}} \frac{\mathcal{M}^{4}}{\mathcal{G} M_{\star} \dot{M}_{\mathrm{disk}}^{2}}\right)^{1 / 7}
$$

where $\bar{c}$ is a dimensionless constant of the order of unity and $\mathcal{M}$ is the stellar magnetic moment. Practically, irrespective of the surface morphology of the stellar field, the magnetic moment can be approximated by the dipole component alone (i.e., $\mathcal{M}=B_{\mathrm{dip}} R_{\star}^{3} / 2$ ) since higher-order harmonics of the field decay rapidly with the radius. For typical young stars, $a_{\text {in }}$ evaluates values not too different from the coronation radius, $a_{\mathrm{co}}=\left(G M_{\star} / \omega_{\star}^{2}\right)^{1 / 3}$. A particular state where the two are set identically equal to each other is referred to as a disk-locked condition (Mohanty \& Shu 2008).

For a given mass accretion rate $\dot{M}$, the amount of added angular momentum depends not only on the launching point, $a_{\mathrm{in}}$ (in this context, the launching radius refers to the point where the accretion flow climbs out of the disk plane to form the accretion column, not to be confused with similar terminology referring to outflows), but also on the structure of the magnetic fields. This dependence arises because in the limit where the magnetic field is strong enough for the associated Lorentz force to be dominant, the system takes on a magnetohydrodynamic force-free state where the cross product of the fluid velocity and magnetic field vanishes (e.g., Moffatt 1978). In other words, the accretion flow follows magnetic field lines so that the field geometry determines, in part, the amount of angular momentum added to the star.

For a given magnetic field configuration, we can construct coordinate systems where one coordinate follows the fields (and hence the flow), while the other two coordinates are orthogonal (following Adams \& Gregory 2012; see also Adams 2011). For tractability we restrict the flow to the poloidal plane, which leads to axisymmetric flow. With this simplification, we can choose the usual azimuthal angle $\varphi$ as the third coordinate. The remaining two coordinates $(p, q)$ depend on the magnetic field geometry. The gradient $\nabla p$ points in the direction of the magnetic field vector, whereas $\nabla q$ is perpendicular so that $\nabla p \cdot \nabla q=0$. We are assuming that the magnetic field is currentfree and curl-free in the region between the inner disk edge and the stellar surface where we are considering the flow. ${ }^{7}$

To start, we take the stellar magnetic field to have both dipole and octupole components, so that the field has the form

$$
\begin{aligned}
\mathbf{B}= & \frac{B_{\mathrm{oct}}}{2 \xi^{5}}\left[\left(5 \cos ^{2} \theta-3\right) \cos \theta \hat{\mathbf{r}}+\frac{3}{4}\left(5 \cos ^{2} \theta-1\right) \sin \theta \hat{\boldsymbol{\theta}}\right] \\
& +\frac{B_{\mathrm{dip}}}{2 \xi^{3}}(2 \cos \theta \hat{\mathbf{r}}+\sin \theta \hat{\boldsymbol{\theta}}),
\end{aligned}
$$

where $\xi=r / R_{\star}$ is the dimensionless radius and $\theta$ is the polar angle in a spherical coordinate system centered on the star. For both the dipole and octupole terms, the leading factors of $1 / 2$ are included so that $B_{\text {dip }}$ and $B_{\text {oct }}$ are the polar strengths of the dipole and octupole components (see also Gregory et al. 2010; Adams \& Gregory 2012). It is convenient to scale out the dipole field strength so that the relative size of the octupole contribution

\footnotetext{
7 Currents exist within the star and most likely within the disk, and act as source terms for the magnetic field. However, strictly speaking, our approximation does not even require the absence of currents in the exterior regions, only that the magnetic field geometry can be written in the forms indicated.
}

is given by the dimensionless parameter

$$
\Gamma \equiv \frac{B_{\mathrm{oct}}}{B_{\mathrm{dip}}}
$$

For T Tauri star-disk systems, observations of magnetic accretion signatures indicate that this parameter typically falls within the range of $0 \leqslant \Gamma \leqslant 10$ (e.g., see Donati et al. 2007, 2008, 2010, 2012, and also Gregory et al. 2010).

For the sake of definiteness, this paper assumes that the dipole and octupole moments are parallel. In some observed $\mathrm{T}$ Tauri systems, however, the magnetic field configurations have octupole moments that are nearly anti-parallel with the dipole moment (i.e., the main positive pole of the octupole is coincident with the negative pole of the dipole). To account for such systems, one can replace $\Gamma$ with $-\Gamma$ (see also Gregory \& Donati 2011), although we leave a full exploration of this issue for future work. For completeness, we also note that the strengths of both components $B_{\text {dip }}$ and $B_{\text {oct }}$, and hence their ratio $\Gamma$, are observed to vary with time for individual stars; as one example, the value of $\Gamma$ for the T Tauri system V2129 Oph was observed to vary by a factor of two over the four year period 2005-2009 (Donati et al. 2011). Although we expect such variations on timescales of $\sim 1 \mathrm{yr}$, over longer timescales the ratio $\Gamma$ does appear to increase with stellar age when all the stars with dipole-octupole fields are considered.

With the magnetic field configuration of Equation (10), the scalar fields that define the coordinate system take the form

$$
\begin{aligned}
& \mathbf{p}=-\frac{1}{4} \xi^{-4} \Gamma\left(5 \cos ^{2} \theta-3\right) \cos \theta-\xi^{-2} \cos \theta \\
& \mathbf{q}=\frac{1}{4} \xi^{-3} \Gamma\left(5 \cos ^{2} \theta-1\right) \sin ^{2} \theta+\xi^{-1} \sin ^{2} \theta
\end{aligned}
$$

Note that the coordinates are dimensionless in this treatment. Note also that for a purely dipole field, $\Gamma \rightarrow 0$, and the coordinates $(p, q)$ simplify to the form (see also Radoski 1967)

$$
\mathbf{p}=-\xi^{-2} \cos \theta \quad \text { and } \quad \mathbf{q}=\xi^{-1} \sin ^{2} \theta .
$$

Let us now consider the field lines that connect the inner disk edge to the stellar surface. To a good approximation, it is sufficient to consider only a single "critical" field line that intersects the fiducial truncation radius of the disk (Ghosh \& Lamb 1978; see also Mohanty \& Shu 2008). At the location of the inner edge of the disk, $a_{\text {in }}$, the angle $\theta=\pi / 2$, and $\xi=\xi_{\text {in }}=a_{\text {in }} / R_{\star}$. The value of the coordinate $q_{0}$ that labels the critical magnetic field line that connects the inner disk edge to the star is thus given by

$$
q_{0}=\frac{1}{\xi_{\text {in }}}\left(1-\frac{\Gamma}{4 \xi_{\text {in }}^{2}}\right)
$$

In the dipole limit, $\Gamma \rightarrow 0$ and $q_{0} \rightarrow 1 / \xi_{\text {in }}$. With the value of $q_{0}$ set by Equation (14), we can evaluate the coordinate at the stellar surface to find the polar angle $\theta_{\star}$ where the magnetic field line (streamline) intercepts the star (where $\xi=1$ ). We thus obtain

$$
q_{0}=(\Gamma+1) \sin ^{2} \theta_{\star}-\frac{5 \Gamma}{4} \sin ^{4} \theta_{\star},
$$

which has the solution

$$
\sin ^{2} \theta_{\star}=\frac{2}{5 \Gamma}\left\{(1+\Gamma) \pm\left[(1+\Gamma)^{2}-5 q_{0} \Gamma\right]^{1 / 2}\right\} .
$$


For most cases of interest, the dipole component of the field dominates at the disk truncation radius, and we must take the minus sign in the above expression. Furthermore, we are interested in cases where $q_{0}$ is small, so the leading order expression becomes

$$
\sin ^{2} \theta_{\star} \approx \frac{q_{0}}{1+\Gamma} \approx \frac{1}{\xi_{\text {in }}(1+\Gamma)} .
$$

With the above results in hand, the specific angular momentum $j$ of a parcel of gas that is accreted along the critical field line is given by the expression

$$
j=n_{\text {in }} R_{\star}^{2} \sin ^{2} \theta_{\star} \approx \frac{n_{\text {in }} R_{\star}^{2}}{\xi_{\text {in }}(1+\Gamma)}=\frac{n_{\text {in }} R_{\star}^{3}}{a_{\text {in }}(1+\Gamma)},
$$

where $n_{\text {in }}=\sqrt{G M_{\star} / a_{\text {in }}^{3}}$ is the (nearly Keplerian) orbital frequency (i.e., mean motion) at the disk truncation radius $a_{\text {in }}$. Due to the rapid falloff of the octupole component of the field with the radius, $n_{\text {in }}$ is nearly the same for systems with pure dipole fields and those with substantial octupole components, and corresponds to the rotation frequency $\omega_{\star}$ of the host star under the disk-locked condition. Thus, as stars increase their octupole component (and hence $\Gamma$ ), the corresponding change in specific angular momentum of the accreted material is produced primarily by changes in $\theta_{\star}$. $^{8}$

Along this line of reasoning, the work of Shu et al. (1994) emphasizes that regardless of the field morphology, the specific angular momentum added to the star is always smaller than that at the launching point in the disk (contrary to what was originally envisioned by Ghosh \& Lamb 1978 in the context of neutron stars). Indeed, while disk winds are responsible for carrying away a relatively small fraction of the mass, they carry away the dominant fraction of angular momentum. At the same time, however, it is important to understand that the specific angular momentum of the accreted material can still be larger than that of the star. This ordering can be seen by explicitly writing out the ratio of the angular momenta, i.e.,

$$
\frac{j_{\text {in }}}{j_{\star}}=\frac{R_{\star}}{(1+\Gamma) I a_{\text {in }}},
$$

where $I$ is the dimensionless momentum of inertia (recall that $I \sim 0.2$ for fully convective stars). As a result, this ratio is often close to unity, so that the accreted material-when acting in isolation - can either spin up or spin down the star.

The presence of $\Gamma$ in the denominators of Equations (18) and (19) plays an important role. Recall that quantitatively, as certain stars evolve and develop stronger octupole components, the factor $\Gamma$ increases from $\sim 0$ to $\sim 10$. As a result, since $j \propto 1 /(1+\Gamma)$, the specific angular momentum of the accreted material decreases by an order of magnitude.

The amount of accreted angular momentum depends sensitively on the launching radius $a_{\text {in }}$. As already mentioned above, for typically observed mass accretion rates where $\dot{M}_{\text {disk }} \sim 10^{-8} M_{\odot} \mathrm{yr}^{-1}$, the truncation radius lies in the range of $a_{\text {in }} \sim 5-15 R_{\star}$. However, disk accretion is known to be highly episodic. If most of the accretion takes place during the brief time intervals when the accretion rates are high, then the value

\footnotetext{
8 We note that the amount of mass accreted by the star is not necessarily affected by the changes in the accretion rate of the angular momentum. This mismatch occurs because a small fraction of the disk's mass is carried away by disk winds (see Figure 1), which also carry away the angular momentum not accreted by the star.
}

of $a_{\text {in }}$ that one should use in the present context for determining the amount of angular momentum added due to disk accretion should be that of the outburst state.

During the outburst phase, the accretion rate can surge up to $\dot{M}_{\text {disk }} \sim 10^{-6} M_{\odot} \mathrm{yr}^{-1}$ (Caratti o Garatti et al. 2011); for extreme cases, such as FU Ori itself, the mass accretion rate can reach $\dot{M}_{\text {disk }} \sim 10^{-5} M_{\odot} \mathrm{yr}^{-1}$. As a result, the truncation radius can move inward by factors of $\sim 4-7$, so that $a_{\text {in }} \sim 1.25-2.5 R_{\star}$. Accordingly, if the truncation radius falls in this range when most of the angular momentum is accreted by the star, then the ratio in Equation (19) varies over the range $(2-4) /(1+\Gamma)$. Thus, a notable amount of angular momentum can be added to the star if the field configuration is dominated by the dipole component, but little angular momentum is added if the octupole dominates.

The spin-up of a star forced by the combined effects of gravitational contraction and accretion of angular momentum is governed by the following differential equation:

$$
\begin{aligned}
& 2 I M_{\star} R_{\star} \omega_{\star} \frac{d R_{\star}}{d t}+I M_{\star} R_{\star}^{2} \frac{d \omega_{\star}}{d t} \\
& =\frac{\omega_{\star}}{(1+\Gamma)} \frac{R_{\star}^{3}}{\left\langle a_{\mathrm{in}}\right\rangle} \frac{M_{\mathrm{disk}}^{0}}{\tau} \frac{1}{(1+t / \tau)^{2}} .
\end{aligned}
$$

It is noteworthy that the above expression is approximate because it uses the smooth, time-averaged mass accretion rate, but assumes that the material is accreted from a closer distance given by the mean value $\left\langle a_{\text {in }}\right\rangle$, which is characteristic of the launching radius for accretion during the outburst state.

In analyzing the behavior of the solution to Equation (20), it is instructive to begin by neglecting the term associated with gravitational contraction. Upon doing so, we obtain the following characteristic timescale for accretion-driven spin-up:

$$
\tau_{\mathrm{accr}}=I(1+\Gamma) \frac{\left\langle a_{\mathrm{in}}\right\rangle}{R_{\star}^{0}} \frac{M_{\star}}{\dot{M}_{\mathrm{disk}}} \sim 40-400 \mathrm{Myr},
$$

where the quoted values correspond to the oft-quoted accretion rate of $\dot{M}_{\text {disk }} \sim 10^{-8} M_{\odot} \mathrm{yr}^{-1}$ and $R_{\star}^{0} /\left\langle a_{\text {in }}\right\rangle \sim 1 / 2$. Note that $\tau_{\text {accr }}$ is substantially longer than a typical disk lifetime. This estimate severely contradicts the notion that the accretion of disk material can act to accelerate a star to a substantial fraction of its breakup velocity in a few Myr (Bouvier 2013). Indeed, the discrepancy arises from the fact that the usual expression for specific angular momentum accretion rate $\dot{j}=a_{\mathrm{in}}^{2} n_{\text {in }}$ (see, e.g., Armitage \& Clarke 1996) overestimates Equation (18) by a factor of $\left(\left\langle a_{\text {in }}\right\rangle / R_{\star}\right)^{3}(1+\Gamma) \gtrsim 10-100$ because it does not properly account for the morphology of the field.

In light of this argument, we expect that the accretion term in Equation (20) will only result in a small correction to the solution (8). For simplicity, let us assume that $\left\langle a_{\text {in }}\right\rangle=$ $\mathcal{X} R_{\star}$, where $\mathcal{X} \sim 2$ is a constant. Under this prescription, Equation (20) is satisfied by the solution

$$
\begin{aligned}
\omega_{\star}= & \omega_{\star}^{0}\left(1+\frac{12 \pi\left(R_{\star}^{0}\right)^{3} \sigma T_{\mathrm{eff}}^{4}}{b G M_{\star}^{2}} t\right)^{2 / 3} \\
& \times \exp \left(\frac{1}{\mathcal{X}(1+\Gamma)} \frac{M_{\mathrm{disk}}^{0}}{M_{\star}} \frac{t}{t+\tau}\right) .
\end{aligned}
$$

Analyzing the solution in the limit $t \gg \tau$ (i.e., when the disk has fully dissipated), we can determine the net result of angular momentum accretion: for an initial disk to star mass ratio of $M_{\text {disk }} / M_{\star}=0.10$, and for stars dominated by dipole fields $(\Gamma=0)$, the rotation rate exceeds the value obtained solely 
from gravitational contraction by about $5 \%$; in contrast, for stars dominated by octupole fields, the rotation rate increases by only about $0.5 \%$. The effects of angular momentum accretion are thus small for dipole systems and negligible for octupole systems.

\subsection{A Comment on Stellar Winds}

Much like the accretion of disk material can add angular momentum to the central star, mass carried away by stellar winds drains angular momentum away from the star. Various models for wind-forced rotational evolution are available in the literature. Notably, a semi-analytical treatment of the winddriven angular momentum loss rate has been formulated by Kawaler (1988). Since its inception, this prescription has been improved, in part with the aid of additional insight gleamed from numerical simulations (see, e.g., Chaboyer et al. 1995; Matt et al. 2012 and the references therein). However, even without actual implementation of the developed models, an upper bound on the angular momentum loss rate can be estimated.

Similarly to the accretionary flow, stellar winds adopt a magnetohydrodynamic force-free profile and travel along magnetic field lines. However, unlike accretion, which takes place along closed field lines, wind must escape the stellar surface along open field lines. An examination of Figure 1 immediately suggests that the polar angles of all field lines along which magnetized wind flows are smaller than that of the critical field line associated with accretion. Aside from this difference, the functional form of wind-driven angular momentum transport is akin to that of the accretionary flow. Consequently, under the disk-locked condition their ratio satisfies

$$
-\left[\frac{d J}{d t}\right]_{\text {wind }}\left[\frac{d J}{d t}\right]_{\mathrm{accr}}^{-1} \lesssim \frac{\dot{M}_{\mathrm{wind}}}{\dot{M}_{\mathrm{accr}}}
$$

Although not perfectly constrained, the typically invoked mass-loss rates due to stellar winds are about an order of magnitude smaller than the typical accretion rates ${ }^{9}$ i.e., $\dot{M}_{\text {wind }} \sim$ $10^{-9} M_{\odot} \mathrm{yr}^{-1}$ (Matt \& Pudritz 2008). As a result, stellar spindown due to angular momentum loss from wind is unlikely to overcome the spin-up that originates from accretion. ${ }^{10}$ Furthermore, recall that we have argued that accretion generally constitutes only a small enhancement to the spin-up associated with gravitational contraction. It is therefore likely that the consequences of stellar wind on rotational evolution can be safely neglected during the disk-bearing phase of the stellar lifetime.

\subsection{Magnetic Star-Disk Coupling}

The final effect we shall consider here is magnetic braking due to star-disk coupling. The understanding of the physical processes inherent to magnetic star-disk interactions has evolved considerably since the original proposal of Königl (1991). However, a detailed description of the model is still unsettled and remains an active area of research (Livio \& Pringle 1992; Armitage \& Clarke 1996; Matt \& Pudritz 2005; Mohanty \& Shu 2008; Zanni \& Ferreira 2009). Here, we shall follow the more or less conventional treatment presented by Matt \& Pudritz (2005) while keeping in mind that various alternatives to this picture exist.

\footnotetext{
9 This notion is expected since magnetized winds during the PMS stage of stellar evolution are thought to be accretion powered, which implies $\dot{M}_{\text {wind }} / \dot{M}_{\text {accr }}<1$.

10 Naturally, an exception to this statement arises if the accretionary flow is quenched by an exclusively open field geometry in the vicinity of $a_{\text {in }}$.
}

The net magnetic torque exerted on the star by the disk is given by the sum of the off-diagonal components of the Maxwell stress tensor, evaluated at $\theta=\pi / 2$ (Livio \& Pringle 1992):

$$
\begin{aligned}
{\left[\frac{d J}{d t}\right]_{\mathrm{mag}} } & =-\frac{4 \pi}{\mu_{0}} \int_{\hat{a}_{\mathrm{in}}}^{\hat{a}_{\mathrm{out}}} B_{\theta} B_{\varphi} a^{2} d a \\
& =-\frac{4 \pi}{\mu_{0}} \int_{\hat{a}_{\mathrm{in}}}^{\hat{a}_{\mathrm{out}}} \gamma \frac{\mathcal{M}^{2}}{a^{4}} d a,
\end{aligned}
$$

where $\hat{a}_{\text {in }}$ and $\hat{a}_{\text {out }}$ represent the inner and outer radii of the magnetically connected region of the disk (i.e., the annulus within which closed magnetic field lines thread the disk) while $\gamma=B_{\varphi} / B_{\theta}$ parameterizes the extent to which field lines are azimuthally twisted by the disk ( $\gamma$ is also referred to as the magnetic pitch angle). The sign of the torque is determined by the differential rotation between the disk and the star: if the disk mean motion is greater than the stellar rotation rate, $[d J / d t]_{\text {mag }}$ is positive, whereas the opposite is true if the disk material trails the unperturbed rotation rate of the field.

The actual magnitude of the azimuthal twist, $\gamma$, depends on the rate at which the field slips back through the disk diffusively. Accordingly, following Matt \& Pudritz (2005), we define a dimensionless diffusivity parameter:

$$
\beta=\frac{\bar{\alpha}}{\mathcal{P}_{\mathrm{m}}} \frac{h}{a},
$$

where $\bar{\alpha}$ is the disk viscosity parameter (Shakura \& Sunyaev 1973), $\mathcal{P}_{\mathrm{m}}$ is the magnetic Prandtl number, and $h$ is the scale height of the disk. As argued by Matt \& Pudritz (2005), any reasonable choice for the diffusivity parameter is bounded from above by $\beta \lesssim 10^{-2}$, an estimate we adopt here.

In terms of $\beta$, the steady-state twist is given by (Uzdensky et al. 2002)

$$
\gamma=\frac{\left(a / a_{\mathrm{co}}\right)^{3 / 2}-1}{\beta} .
$$

It is important to understand that magnetic field lines cannot stretch indefinitely. As shown by Uzdensky et al. (2002), beyond a critical twist of the order of unity, the topology of the magnetic field transitions from closed to open field lines. Thus, Equation (26) implicitly defines critical values $\hat{a}_{\text {in }}$ and $\hat{a}_{\text {out }}$ as the roots when $|\gamma|=1$. Clearly, for our adopted value of $\beta$, the magnetically connected region within the disk is quite narrow, with the transition radii not deviating away from the corotation radius by more than $\sim 1 \%$.

In light of the dependence of the twist on the distance away from the corotation radius (Equation (26)), it is apparent that the magnitude of the total magnetic torque (Equation (24)) is predominately controlled by the exact value of $a_{\text {in }}$. That is, if the disk is truncated beyond the outer field transition radius $\left(\hat{a}_{\text {out }}<a_{\text {in }}\right.$ ), then the magnitude of the magnetic torque is null. ${ }^{11}$ If the disk is truncated interior to the inner transition radius $\left(a_{\text {in }}<\hat{a}_{\text {in }}\right)$, then the spin-down torques, which arise from the part of the disk beyond the corotation radius, are to first order canceled out by the spin-up torques that arise from the part of the disk interior to the corotation radius. Consequently, in this case, it can be expected that $[d J / d t]_{\mathrm{mag}} \propto \beta^{2}$. Magnetic braking is maximized if the disk truncation radius corresponds exactly to the corotation radius, i.e., the disk-locked condition

\footnotetext{
11 Note that in this case, magnetically controlled accretion would also cease (Matt \& Pudritz 2005).
} 
$\left(\hat{a}_{\text {in }}=a_{\text {in }}\right)$. In this case, we expect that $[d J / d t]_{\text {mag }} \propto \beta$. Note that this picture is considerably less optimistic than the one envisioned by Armitage \& Clarke (1996), where the field was taken to couple to the entire disk.

The rates of angular momentum transport in the three regimes are summarized as follows:

$$
\left[\frac{d J}{d t}\right]_{\mathrm{mag}}= \begin{cases}0 & \left(a_{\text {in }}>\hat{a}_{\text {out }}\right) \\ -\frac{4 \pi}{3 \mu_{0}} \frac{\beta \mathcal{M}^{2}}{a_{\mathrm{co}}(1+\beta)^{2}} & \left(a_{\text {in }}=a_{\mathrm{co}}\right) \\ -\frac{16 \pi}{3 \mu_{0}} \frac{\beta^{2} \mathcal{M}^{2}}{a_{\mathrm{co}}\left(1-\beta^{2}\right)^{2}} & \left(a_{\text {in }}<\hat{a}_{\text {in }}\right) .\end{cases}
$$

Similarly, we can evaluate the corresponding spin-down timescales:

$$
\tau_{\mathrm{mag}}=\left\{\begin{array}{l}
\infty \\
\frac{3 I}{4 \pi \omega_{\star}^{0}} \frac{(1+\beta)^{2}}{\beta}\left(\frac{G M^{2} R^{2} \mu_{0}}{\mu^{2}}\right) \sim 3 \mathrm{Myr} \\
\frac{3 I}{16 \pi \omega_{\star}^{0}} \frac{\left(1-\beta^{2}\right)^{2}}{\beta^{2}}\left(\frac{G M^{2} R^{2} \mu_{0}}{\mu^{2}}\right) \sim 100 \mathrm{Myr},
\end{array}\right.
$$

where the vertical ordering is identical to Equations (27). It is noteworthy that the terms inside the parentheses in Equations (28) approximately correspond to the ratio of hydrostatic pressure in the stellar interior and magnetic pressure at the stellar surface.

From these estimates, we can expect that for values of $a_{\mathrm{in}}$, chosen in a non-pathological manner, magnetic braking may be competitive with spin-up due to accretion but not with spinup due to gravitational contraction. An exception to this statement arises when the disk-locked condition is imposed (albeit in an ad hoc way), and the magnetic braking timescale approaches the same order of magnitude as the Kelvin-Helmholtz time. Although the disk-locked condition itself is an idealization due to the episodic nature of accretion, it may be argued that $a_{\text {in }}$ periodically sweeps across $a_{\mathrm{co}}$, yielding brief (but possibly frequent) spurts of efficient magnetic braking. Consequently, the extent to which angular momentum exchange via magnetic disk-star coupling is responsible for the observed rotational distribution of $\mathrm{T}$ Tauri stars may be subject to the detailed nature of time-variability inherent to accretion. A thorough exploration of this issue is beyond the scope of this paper.

\subsection{Parameterized Rotational Evolution}

With the relevance of the individual angular momentum transfer mechanisms identified, we shall now perform a few exemplary numerical calculations of the post-embedded rotational evolution of a Sun-like star during its disk-bearing phase. Specifically, we integrate a variant of Equation (20) including the effects of accretion, time evolution of the stellar radius, and external magnetic torques given by Equations (27). Stellar and disk parameters are taken as before and the magnetic moment is assumed to correspond to a $1.5 \mathrm{kG}$ surface field at $t=0$ $\left(R_{\star}=R_{\star}^{0}\right)$. As initial stellar rotation rates, following Gallet $\&$ Bouvier (2013), we adopt $P_{\star}^{0}=2,7$, and 10 days, which corresponds to characteristic fast, median, and slow rotators, as dictated by the observed distribution.

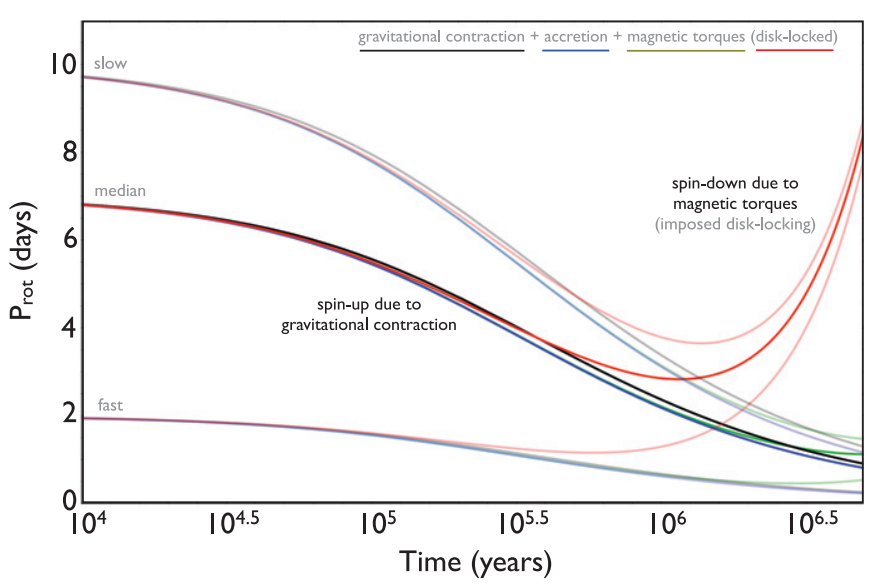

Figure 3. Rotational evolution of fully convective $(n=3 / 2)$ stars including stellar contraction, accretion of angular momentum from a disk, as well as magnetic braking. The time evolution of the stellar period is shown for three different initial conditions: $P_{\star}^{0}=2,7$, and 10 days, corresponding to characteristic fast, median, and slow rotators, as dictated by the observed distribution. The final rotational distribution of PMS stars appears to be dictated predominantly by the extent to which the disk truncation radius corresponds to the stellar corotation radius, as this is the primary parameter that determines the effectiveness of magnetic braking. If the truncation radius significantly deviates away from the corotation radius, gravitational contraction dominates the rotational evolution, leading to stellar spin-up.

(A color version of this figure is available in the online journal.)

The evolutionary tracks are shown in Figure 3. For each initial condition, four solutions are presented. The black lines denote isolated spin up due to gravitational contraction (these solutions also correspond to accreting stars, whose fields are dominated by the octupole component, i.e., $\Gamma=10$ ). The blue lines are solutions that account for accretion in a dipolar-dominated $\operatorname{system}(\Gamma=0)$. Solutions that additionally account for magnetic braking in the case of $a_{\text {in }}<\hat{a}_{\text {in }}$ are shown with the green lines, while the red lines correspond to solutions that adopt a magnetic torque with the disk-locked condition.

As expected, the spin-up due to the reduction in the moment of inertia associated with gravitational contraction dominates the early stages of rotational evolution. Although visible, the effects of accretion and magnetic braking (without assuming the disklocking condition) clearly contribute only small corrections to the isolated solution. However, under the assumption of disklocking, magnetic torques dominate the later stages of evolution and effectively de-spin the stars to rotational periods on the order of the median observed value (Affer et al. 2013). It is noteworthy that the dominance of magnetic torques increases with time. This is a direct consequence of gravitational contraction: as the stellar radius decreases, so does the ratio of interior hydrostatic to surface magnetic pressures, facilitating more efficient braking.

It is interesting to note that whether or not the disk-locking condition is imposed on the magnetic torques, the variance of the final (evolved) rotation rates is diminished compared to the spread in initial conditions. Furthermore, the rotation rates corresponding to isolated gravitational contraction and those corresponding to magnetically spun-down stars appear to approximately bracket the observed distribution. Thus, if our results are taken at face value, it may be argued that the presence of both slow and fast rotators in the observational sample is primarily a consequence of the magnetic torque's sensitive dependence on the truncation radius, leading to variable coupling patterns among different stars. Note also that the characteristic timescale for the evolution of the stellar spin is on the order of a few Myr. 
This suggests that the timescales for rotational evolution (Figure 3), planet formation (Pollack et al. 1996) and disk removal (e.g., Hernández et al. 2007) are all roughly comparable.

Despite the apparent points of agreement between the above calculations and the observed distribution, it should be kept in mind that our treatment contains a number of approximations. Most notably, our calculation of magnetic braking is idealized and the extent to which the analogy between the corotation radius and the truncation radius is relevant remains elusive. As already discussed above, this issue directly ties into the fact that our treatment uses time-averaged quantities to model the accretion flow, which is fundamentally episodic. This means that the parameter values that should be used are somewhat uncertain and will vary from system to system. Secular changes in the controlling quantities also cannot be ruled out. A much less dominant, but nevertheless important point is that in our calculations, the amount of accreted angular momentum is overestimated (by a small amount) because we assume that all of the disk material lands on the star. In actuality, somewhere between a third and a tenth of the disk mass is carried away by winds that are launched in conjunction with the accretion flow (Shu et al. 1994), while additional stellar braking is accomplished through stellar winds (see Figure 1). Finally, the results shown here use the dimensionless moment of inertia, $I=0.20$, appropriate for a fully convective star; the moment of inertia could be smaller if the star develops a radiative core or if only the outer layers are rotating (and smaller I would amplify the effects of angular momentum accretion).

In spite of the uncertainties, the characteristic behavior depicted in Figure 3 suggests the following general features for the time dependence of stellar spin. During the early epochs of classical star-disk evolution, gravitational contraction unambiguously leads to stellar spin-up. Subsequently, stars are spun down (to a variable extent) by torques associated with magnetic star-disk coupling. The final distribution of rotation rates obtained within the context of our calculations appear to be largely independent of initial conditions. This means that even if stars typically emerge from the embedded, protostellar stage as fast rotators, we expect the combined effects of disk-locking and winds/outflows to successfully brake stellar rotation from near-breakup rates to periods on the order of $P_{0} \sim 7$ days (Shu et al. 1994; Herbst et al. 2002, 2007) during the diskbearing phase. Although the tracks in Figure 3 are plotted with a single set of assumed controlling parameters, it is implicitly understood that the range of such parameters can be large, and the substantial breadth of the PMS stellar rotational distribution appears to be consistent with the calculations presented above. Moreover, additional modulation due to the accretion of disk angular momentum and perhaps other torques will surely act to further homogenize the distribution.

\section{DYNAMICS OF THE STELLAR SPIN AXIS}

Having characterized PMS stellar rotational evolution due to gravitational contraction, accretion of disk material, and magnetic braking in the previous section, we now turn our attention to the orientation of stellar spin axes with respect to planetary orbits. Specifically, the aim of the following calculation is to construct a simple quantitative model for the spin-axis dynamics of a rotating star, forced by gravitational interactions as well as magnetically controlled accretion arising from a azimuthally symmetric circumstellar disk.

Generally, it is expected that the precession timescale of the stellar pole will greatly exceed both the stellar rotation period and the orbital period of fluid parcels within the disk. As a result, here we can work within the context of orbit-averaged perturbation theory (Murray \& Dermott 2000). To the extent that a rotating star can be represented as an oblate spheroid, the dynamics of its spin-pole can be approximated as those of a point mass surrounded by an orbiting ring with mass

$$
\tilde{m}=\left[\frac{3 M_{\star}^{2} \omega^{2} R_{\star}^{3} I^{4}}{4 \mathcal{G} k_{2}}\right]^{1 / 3},
$$

and semimajor axis

$$
\tilde{a}=\left[\frac{16 \omega^{2} k_{2}^{2} R_{\star}^{6}}{9 I^{2} \mathcal{G} M_{\star}}\right]^{1 / 3} .
$$

To avoid confusion, we adopt a notation where the quantities corresponding to the above-defined ring are marked by tildes, while the analogous quantities referring to the disk are marked by primes.

Taking into account the fact that the star is orbited by a massive protoplanetary disk, the stellar ring will not remain stationary (Binney \& Tremaine 1987). Specifically, its longitude of ascending nodes will recess. Away from elliptical fixed points in phase space, such a recession may give rise to associated changes in the inclination. Non-trivial evolution of the stellar spin-pole is further ensured if the orbital properties of the disk evolve in time. To a satisfactory approximation, the dynamical evolution of the stellar spin axis can be calculated within the framework of a modified Laplace-Lagrange secular theory (see, e.g., Murray \& Dermott 2000; Morbidelli 2002).

It can be trivially shown that angular momenta of protoplanetary disks (and by extension, planetary systems) greatly exceed that of even the most rapidly rotating stars. As a result, it is sensible to calculate the evolution of the stellar spin axis within the framework of a restricted secular treatment. In other words, we can neglect the back reaction of the changes in the stellar spin axis on the inclination dynamics of the disk. Provided that self-gravity of the disk is typically sufficiently strong to ensure that the disk behaves as an effectively rigid body (see Batygin et al. 2011; Morbidelli et al. 2012), the extent of warping within the disk should be negligible, and this restricted assumption is likely to be well satisfied for any relevant choice of parameters.

To begin with, consider the dynamical evolution of the stellar spin pole due to a massive ring of infinitesimal radial extent, $d a^{\prime}$. As a starting approximation, we consider the physical parameters of the star and the disk (i.e., the stellar radius, rotation rate, moment of inertia, disk mass, etc.) to be constant. This assumption will be lifted later. To the leading order in inclination, $i$, the Hamiltonian that governs the dynamics of the stellar rotation pole (Murray \& Dermott 2000) is

$$
\begin{aligned}
d \mathcal{H}= & -\frac{\mathcal{G} \tilde{m} d m^{\prime}}{a^{\prime}}\left[f \sin ^{2}\left(\frac{\tilde{i}}{2}\right)\right. \\
& \left.-2 f \sin \left(\frac{\tilde{i}}{2}\right) \sin \left(\frac{i^{\prime}}{2}\right) \cos \left(\tilde{\Omega}-\Omega^{\prime}\right)\right] .
\end{aligned}
$$

Here, the quantity $d m^{\prime}=\left(2 \pi \Sigma a^{\prime} d a^{\prime}\right)$ is the mass of the ring (where $\Sigma \propto r^{-1}$ is the disk surface density), $\Omega$ is the longitude of the ascending node, while $f$ is a constant that depends only on the semimajor axes $a$.

It is important to understand that this Hamiltonian only provides a leading order approximation to the true dynamics 
of the system. The solution becomes increasingly imprecise as the mutual inclination between the stellar spin axis and the orbital plane of the disk is increased. As a result, in the vicinity of linear secular resonance, where the characteristic precession timescale of the star matches the torquing timescale of the disk, any solution obtained from the above Hamiltonian will grossly overestimate the amplitude of oscillations. ${ }^{12}$ That said, away from the near-resonant domain, this flavor of secular theory has been shown to be a surprisingly good approximation to higherorder secular solutions ${ }^{13}$ (Van Laerhoven \& Greenberg 2012).

As stated above, it is sensible to assume that the inner edge of the disk, $a_{\mathrm{in}}^{\prime}$, is truncated at or near the stellar corotation radius, meaning $n_{\text {in }}^{\prime} \simeq \omega_{\star}$. With this constraint in mind, it can be easily checked that for any reasonable set of parameters, $\alpha \equiv \tilde{a} / a_{\text {in }}^{\prime} \ll 1$. Taking advantage of the smallness of $\alpha$, the expression for $f$ can be simplified considerably. More specifically, after expressing the Laplace coefficient of the first kind $b_{3 / 2}^{(1)}(\alpha)$ (see Chapter 7 of Murray \& Dermott 2000) as a hypergeometric series, we can make the approximation $b_{3 / 2}^{(1)}(\alpha) \simeq 3 \alpha$. As a result, the coefficient $f$ can be expressed in the form

$$
f=-\frac{3}{2}\left(\frac{\tilde{a}}{a^{\prime}}\right)^{2}
$$

Because Keplerian orbital elements do not constitute a canonically conjugated set, we must change variables before proceeding further. Specifically, let us convert to modified Poincaré action-angle coordinates:

$$
\begin{aligned}
\tilde{Z} & =(1-\cos (\tilde{i})) & \tilde{z} & =-\tilde{\Omega} \\
Z^{\prime} & =\left(1-\cos \left(i^{\prime}\right)\right) & & z^{\prime}=-\Omega^{\prime} .
\end{aligned}
$$

Note that compared with the usual form of Poincaré coordinates (see Chapter 2 of Murray \& Dermott 2000), the above actions have been reduced by a factor of $\Lambda=m \sqrt{G M_{\star} a}$. In order to consider the above variables as canonical (keeping in mind that $\left(Z^{\prime}, z^{\prime}\right)$ are pre-determined functions of time), we must scale the Hamiltonian by a factor of $\tilde{m} \sqrt{G M_{\star} \tilde{a}}$. Utilizing the half-angle formula, we obtain the following expression for $d \mathcal{H}$ :

$$
d \mathcal{H}=\frac{3 \pi \mathcal{G} \Sigma d a^{\prime}}{2 \sqrt{\mathcal{G} M_{\star} \tilde{a}}}\left(\frac{\tilde{a}}{a^{\prime}}\right)^{2}\left[\tilde{Z}-2 \sqrt{\tilde{Z}} \sqrt{Z^{\prime}} \cos \left(\tilde{z}-z^{\prime}\right)\right] .
$$

As stated above, the Hamiltonian (34) dictates the dynamical evolution of the stellar spin axis due to an infinitesimal ring of mass. In order to obtain a Hamiltonian that accounts for the forcing that arises from the entire disk, we must integrate $d \mathcal{H}$ radially with respect to $d a^{\prime}$. Noting that the disk mass has the form

$$
M_{\mathrm{disk}}=2 \pi \int_{a_{\mathrm{in}}^{\prime}}^{a_{\mathrm{out}}^{\prime}} a^{\prime} \Sigma_{0}\left(\frac{a_{0}^{\prime}}{a^{\prime}}\right) d a^{\prime} \simeq 2 \pi \Sigma_{0} a_{0}^{\prime} a_{\mathrm{out}}^{\prime},
$$

where $\Sigma_{0}$ is the surface density at $a_{0}^{\prime}$, we define the characteristic spin-axis precession frequency $\mathcal{F}$ according to

$$
\begin{aligned}
\mathcal{F}= & \int_{a_{\text {in }}^{\prime}}^{a_{\text {out }}^{\prime}} \frac{3 \pi \mathcal{G} \Sigma_{0} a_{0}}{2 \sqrt{\mathcal{G} M_{\star} \tilde{a}} a^{\prime}}\left(\frac{\tilde{a}}{a^{\prime}}\right)^{2} d a^{\prime}=\frac{3}{8} \frac{2 \pi \mathcal{G} \Sigma_{0} a_{0}^{\prime} \tilde{a}^{2}}{\sqrt{\mathcal{G} M_{\star} \tilde{a}}} \\
& \times\left(\frac{1}{\left(a_{\text {in }}^{\prime}\right)^{2}}-\frac{1}{\left(a_{\text {out }}^{\prime}\right)^{2}}\right) \simeq \omega_{\star} \frac{3}{8}\left(\frac{\omega_{\star}}{\tilde{n}} \frac{M_{\text {disk }}}{M_{\star}} \frac{a_{\text {in }}^{\prime}}{a_{\text {out }}^{\prime}}\right) .
\end{aligned}
$$

\footnotetext{
12 In fact, at exact linear resonance, the solution encounters an unphysical singularity that cannot be removed by a change of variables.

13 Although, we note that such correspondence is not assured generally.
}

In this work, we adopt $a_{\text {out }}^{\prime}=50 \mathrm{AU}$ as a characteristic size of a typical protoplanetary disk (e.g., Anderson et al. 2013). With these specifications, the Hamiltonian takes on a simple form:

$$
\mathcal{H}=\mathcal{F}\left[\tilde{Z}-2 \sqrt{\tilde{Z}} \sqrt{Z^{\prime}} \cos \left(\tilde{z}-z^{\prime}\right)\right]
$$

After prescribing a time-dependence for the dynamical state of the disk, the above Hamiltonian constitutes a system with 1.5 degrees of freedom. In the regime of interest, however, the system can be reduced to a single degree of freedom through an appropriate choice of the coordinate system's orientation.

As shown by Batygin (2012), the longitude of the ascending node and orbital inclination, as measured in a frame coplanar with the protoplanetary disk at $t=0$, undergoes, respectively, quasi-linear and cycloidal oscillations in widely separated binary stellar systems. Indeed, such a frame represents the spinpole orientation of a non-precessing (i.e., purely spherical) star that is initially in perfect alignment with the disk. Consequently, the oscillatory behavior of the disk's inclination was used to argue for primordial misalignments between hot Jupiter orbits and their host stars. However, in a reference frame that is coplanar with the orbit of the stellar companion, the disk inclination (that is initially not null) is approximately preserved and the recession rate of the disk's ascending node is constant. In such a reference frame, we may write $z^{\prime}=v t$, where $v$ is the disk-torquing frequency and $Z^{\prime}=Z_{0}^{\prime}$.

To make the necessary simplification, we extend the phase space to four dimensions by adding an action $\mathcal{T}$, conjugated to $t$, thereby obtaining an autonomous Hamiltonian:

$$
\mathcal{H}=\mathcal{F}\left[\tilde{Z}-2 \sqrt{\tilde{Z}} \sqrt{Z_{0}^{\prime}} \cos (\tilde{z}-v t)\right]+\mathcal{T} .
$$

Next, we perform a canonical change of variables given by the following generating function of the second kind:

$$
G_{2}=(\tilde{z}-v t) \Theta+t \Xi .
$$

Upon application of the transformation equations, we obtain

$$
\begin{aligned}
& \tilde{Z}=\partial G_{2} / \partial \tilde{z}=\Theta \quad \theta=\tilde{z}-v t \\
& \mathcal{T}=\partial G_{2} / \partial t=-v \tilde{Z}+\Xi \quad \zeta=t
\end{aligned}
$$

The physical meaning behind the transformation lies in transferring to a reference frame that is aligned with the orbital plane of the binary companion but co-precesses with the ascending node of the disk. An advantage of the new coordinates is that the perfectly aligned state is always represented by the point $(\Theta, \theta)=\left(Z_{0}^{\prime}, 0\right)$. Furthermore, after the transformation, the angle $\zeta$ is absent from $\mathcal{H}$, rendering $\Xi$ a constant of motion that we subsequently drop. The Hamiltonian is now transformed into a system with a single degree of freedom:

$$
\mathcal{H}=\mathcal{F}\left[\Theta-2 \sqrt{Z_{0}^{\prime}} \sqrt{\Theta} \cos (\theta)\right]-v \Theta .
$$

At this point, the Hamiltonian cannot be simplified further. However, the action-angle coordinates described above posses a coordinate singularity at $\Theta=0$, which can be removed by converting to Cartesian coordinates:

$$
x=\sqrt{2 \Theta} \cos (\theta) \text { and } y=\sqrt{2 \Theta} \sin (\theta) .
$$

The equations of motion can be written down more succinctly by defining a single complex variable

$$
\mu=\frac{x+\imath y}{\sqrt{2}}=\sqrt{\Theta} \mathbf{e}^{\imath \theta},
$$


where $\imath=\sqrt{-1}$. With this definition, the Hamiltonian now takes the form

$$
\mathcal{H}=\mathcal{F}\left[\mu \mu^{*}-\sqrt{Z_{0}^{\prime}}\left(\mu+\mu^{*}\right)\right]-v \mu \mu^{*} .
$$

To motivate one final transformation, let us recall that the misalignment angle of interest is not measured in a frame aligned with the binary orbit (where the angle is given by $\mu$ ), but rather one associated with the planetary orbit. We thus perform a canonical translation into a disk-aligned frame through the definition

$$
\eta \equiv \mu-\sqrt{Z_{0}^{\prime}}
$$

After dropping constant terms, this substitution transforms the Hamiltonian into

$$
\mathcal{H}=\mathcal{F} \eta \eta^{*}-v\left[\eta \eta^{*}+\sqrt{Z_{0}^{\prime}}\left(\eta+\eta^{*}\right)\right]
$$

The Hamiltonian (46) governs the conservative component of disk-star interactions. In certain circumstances, it may be argued that this expression does not provide a full evolutionary description. For example, in Section 3, we saw that accretion of disk material onto the stellar surface and magnetic disk-star coupling can result in limited transfer of angular momentum. Consequently, in systems where the orientations of the stellar spin axis and the disk's orbital plane are not aligned, dissipative processes can in principle provide corrections to a solution obtained exclusively from Equation (46).

On a related note, it is important to understand that within the context of stellar spin-axis dynamics, the role of gravitational contraction (which may dominate stellar spin-up) is not analogous to that of accretion or magnetic braking, because the former is a processes that conserves angular momentum (and would operate similarly in absence of the disk). In other words, gravitational contraction itself neither leads to excitation nor damping of mutual star-disk inclination. ${ }^{14}$ However, there exists an indirect effect associated with gravitational contraction: as stellar radius decreases and the spin is modulated, the coefficient of the Hamiltonian (46) will also vary in accord with Equations (30) and (36). As will be shown below, this plays a central role in determining if the aforementioned encounter with a secular resonance occurs.

Generally, the details of magnetically controlled disk-star interactions are rather complex and can only be faithfully captured within the framework of numerical MHD simulations (see Romanova et al. 2002, 2003). However, as shown in Section 3, the characteristic timescale of angular momentum transfer can be estimated analytically. For the purposes of our calculations, it is sensible to adopt such estimates instead of performing fullfledged numerical MHD simulations. Consequently, here we model the accretion-driven re-alignment of the star as an exponential decay of the mutual inclination angle and adopt $\tau_{\text {accr }}$ (as given by Equation (21)) as the $e$-folding time. We note, however, that the inclusion of the accretion term is done solely for generality as its practical effect is negligible.

Evolution of the spin-pole orientation facilitated by magnetic torques is considerably more complicated. Naively, it is tempting to adopt the same functional form for this process

\footnotetext{
14 In principle, a similar argument applies to stellar winds if the wind profile (i.e., magnetic field geometry) possesses cylindrical symmetry around the stellar spin axis.
}

as that due to accretion. However, realistically this may not be appropriate, since as shown by Lai et al. (2011; see also Jackson 1998) magnetic torques can act to excite, rather than damp mutual star-disk inclination. Furthermore, when acting in conjunction with accretion, magnetic torques may act to evolve the star-disk misalignments toward equilibrium values that sensitively depend on the details of the system at hand. In light of the uncertainties inherent to the physics of magnetic tilting, we shall neglect this process in our model. Indeed, the notion that magnetic tilting appears to be competitive with accretion (Lai et al. 2011) renders our disregard for its effects reasonable, since the latter operates on timescales somewhat longer than typical lifetimes of protoplanetary disks (see Equation (21)). Still we note that our model may represent a conservative perspective on primordial star-disk misalignments.

Putting all of the above pieces together, we find that the evolution of the inclination vector is governed by the differential equation

$$
\begin{aligned}
\frac{d \eta}{d t} & =\left[\imath \frac{\partial \mathcal{H}}{\partial \eta^{*}}\right]+\left[\frac{d \eta}{d t}\right]_{\mathrm{accr}} \\
& =\imath(\mathcal{F}-v) \eta-\imath v \sqrt{Z_{0}^{\prime}}-\frac{\eta}{\tau_{\mathrm{accr}}} .
\end{aligned}
$$

The solution is characterized by three physical timescales: the stellar spin-axis precession timescale $2 \pi / \mathcal{F}$, the disk torquing timescale $2 \pi / \nu$, and the accretion timescale $\tau_{\text {accr }}$. Of course, in reality, all three of these characteristic timescales are themselves time dependent and evolve throughout the lifetime of the disk. Specifically, both the spin-axis precession and accretion timescales are determined by evolving physical properties of the star and the disk, whereas the disk-torquing timescale is sensitive to changes in the perturbing companion's orbit that may arise from the binary's evolution within its birth cluster or external perturbations from passing stars. In spite of these complications, it is instructive to examine the limiting cases of the solution analytically.

Assuming a perfectly aligned initial condition $\eta_{0}=0$, as well as constant values for $\mathcal{F}, v$, and $\tau_{\text {accr }}$, Equation (47) can be integrated to take the form

$$
\eta=\sqrt{Z_{0}^{\prime}}\left[\frac{\mathbf{e}^{l(\mathcal{F}-v) t-t / \tau_{\text {acc }}}-1}{1-\imath /\left(\nu \tau_{\text {accr }}\right)-F / v}\right] .
$$

Upon examination of the solution, we can identify four characteristic regimes, which we list below. For reference, a typical representation of each regime is depicted in Figure 4.

Accretion-dominated regime. Arguably, the simplest dynamical picture is one where the disk-torquing timescale is exceptionally long, and accretion dominates. This occurs in the limit $1 /\left(v \tau_{\text {accr }}\right) \gg(1, \mathcal{F} / v)$. Accordingly, the denominator in Equation (48) retains only the accretion term, while the exponential in the numerator vanishes over a comparatively short timescale. Independent of the particular initial condition, the (diminutive) value of the misalignment angle is controlled entirely by the smallness of $\nu \tau_{\text {accr }}$ and the solution reduces to the form

$$
\eta^{(\mathrm{accr})} \rightarrow \imath \sqrt{Z_{0}^{\prime}}\left(\nu \tau_{\mathrm{accr}}\right) \simeq 0
$$

This regime is characteristic of very young (embedded) systems where the disk is massive and the accretion rate is maximal, ensuring that any excitation of the misalignment angle arising from external perturbations of the protoplanetary disk damps 


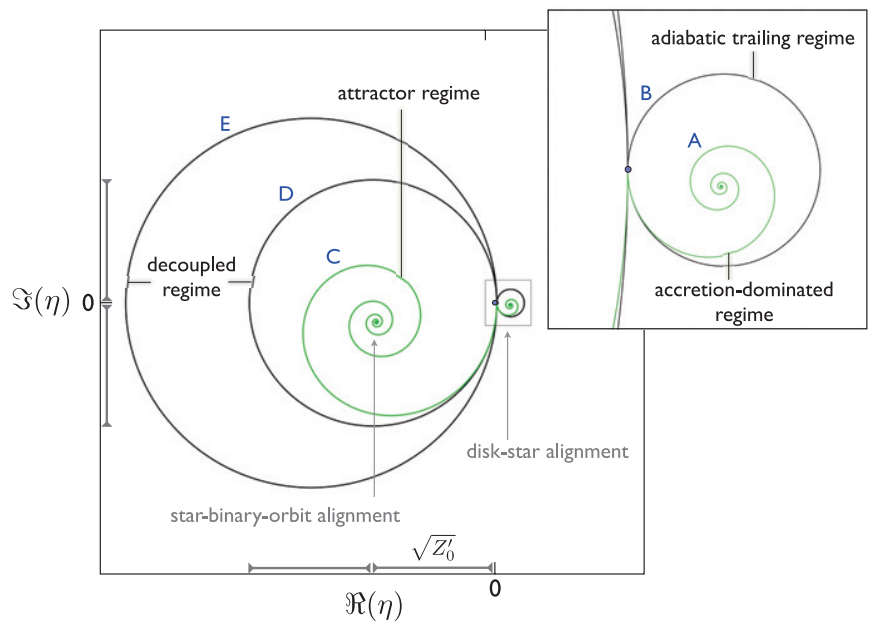

Figure 4. Phase-space representation of the characteristic regimes of stellar spinaxis dynamics (as dictated by Equation (48)). The green curves represent regimes where accretion plays an appreciable role, while the black curves depict regimes where accretion is negligible. The origin corresponds to a disk-aligned state of the star, which also corresponds to the adopted initial condition. Recalling the variable transformations utilized in the derivation of Equation (48), the misalignment angle is obtained by the distance from the origin to the point on the orbit at which the system resides at a time $t$, i.e., $\psi=\arccos \left(1-\eta \eta^{*}\right)$. The disk inclination, encapsulated into $\sqrt{Z_{0}^{\prime}}$, which also sets the scale of the orbits, is shown with the gray lines.

(A color version of this figure is available in the online journal.)

away rapidly. A phase-space representation of an accretiondominated solution is shown as a green spiral, labeled $\mathrm{A}$ in the small inset of Figure 4. Here, the phase-space trajectory spirals into the origin.

Adiabatic trailing regime. Another extreme parameter regime that prevents significant excitation of misalignment is one where efficient disk-star angular momentum transfer is accomplished through gravitational coupling. For the sake of definitiveness, suppose that accretion is completely negligible (i.e., $\tau_{\text {accr }}=\infty$ ) but the spin-axis precession timescale is much shorter than the external torquing timescale, so that $\mathcal{F} / v \gg 1$. In this regime, we obtain an oscillatory solution, where the amplitude is small, $|\eta| \sim v / \mathcal{F}$, and the solution approaches the form:

$$
\eta^{(\mathrm{ad})} \rightarrow \sqrt{Z_{0}^{\prime}}\left(\frac{v}{\mathcal{F}}\right)\left[1-\mathbf{e}^{l \mathcal{F} t}\right] \simeq 0
$$

In the inset of Figure 4, this adiabatic-trailing regime is shown with a black (circular) orbit, labeled B. This type of behavior is characteristic of systems where the stars are spun up at early times; the stars can then develop a strong gravitational quadrupole moment, which facilitates gravitational coupling between the star and the disk. We note that this regime may also be relevant for systems where the gravitational torquing of the disk is exceptionally slow (e.g., when the disk has a small radius, or the binary companion has a wide orbit), so that even a modestly deformed star can keep up with the twist.

Attractor regime. Now we consider a system that does not satisfy either of the above criteria. For simplicity, imagine a perfectly spherical $(\mathcal{F}=0)$, accreting star, whose re-alignment timescale is not overwhelmingly longer than the disk-torquing timescale, i.e., $v \tau_{\text {accr }} \sim 5-10$. In this case, over a few disktorquing times, the exponential in Equation (48) decays away while the denominator is approximately unity. The solution in

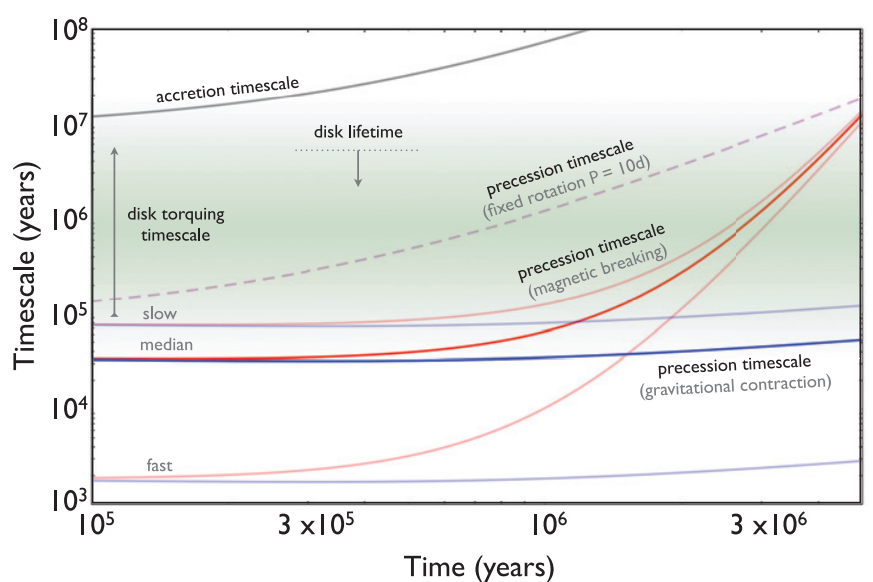

Figure 5. Timescales associated with the dynamics of the stellar spin axis, given here as a function of time. The blue and red curves represent the precession timescale, $2 \pi / \mathcal{F}$, and correspond to the rotational evolution sequences depicted in Figure 3. Accordingly, the blue curves are those where the star is subjected exclusively to gravitational contraction and accretion while the red curves are those where magnetic braking successfully spins the star down to periods on the order of $\sim 10$ days in $\sim 5$ Myr. The precession timescale corresponding to a star whose rotational period is held fixed at $P_{\star}=10$ days is shown as a purple dashed curve. The accretion timescale is shown as a gray curve, whereas typical disk-torquing timescales are represented by a green shade.

(A color version of this figure is available in the online journal.)

this regime reduces to the form

$$
\eta^{(\mathrm{att})} \rightarrow-\sqrt{Z_{0}^{\prime}}
$$

An orbit of this type is shown in Figure 4 as a big green spiral labeled $\mathrm{C}$. The physical meaning of this solution is most easily understood by recalling the variable transformation of Equation (45). In the frame aligned with the binary orbit, the above limit corresponds to a null inclination, $\mu=0$, which represents alignment between the angular momentum vectors of the binary orbit and the stellar spin. Naturally, in this regime, the star-disk misalignment angle becomes identically equal to the misalignment angle between the primordial disk and the binary orbit. Intuitively, this end state is sensible because it corresponds to an equilibrium, reached upon phase-averaging the angular dependence of accretion from a rapidly torqued disk.

Decoupled regime. Finally, we consider the regime where the timescales associated with precession of the stellar spin axis and accretion are both much longer than the disk-torquing timescale, implying that $\nu \tau_{\text {accr }} \gg 1$ and $\mathcal{F} / v \ll 1$. In this case, the denominator in Equation (48) is (again) approximately unity, while the accretion term in the exponential can be dropped, yielding a solution of the form

$$
\eta^{(\mathrm{dec})} \rightarrow \sqrt{Z_{0}^{\prime}}\left[\mathbf{e}^{l(\mathcal{F}-v) t}-1\right]
$$

It can be shown that in terms of the original $(\tilde{Z}, \tilde{z})$ variables from Equation (33), this solution corresponds to a state where the star remains at its initial condition for all time. The phasespace trajectories associated with this solution are shown as black curves in Figure 4, labeled $\mathrm{D}$ (with $\mathcal{F} / v=0$ ) and $\mathrm{E}$ (with $\mathcal{F} / v=1 / 3$ ). Indeed, the non-triviality of the above limit arises entirely from fixing the inclination and longitude of the ascending node of the reference frame to coincide to that of the disk at all times. As shown by Batygin (2012) through an alternative derivation, in this regime the disk-star misalignment angle can be represented parametrically by a cycloid. 

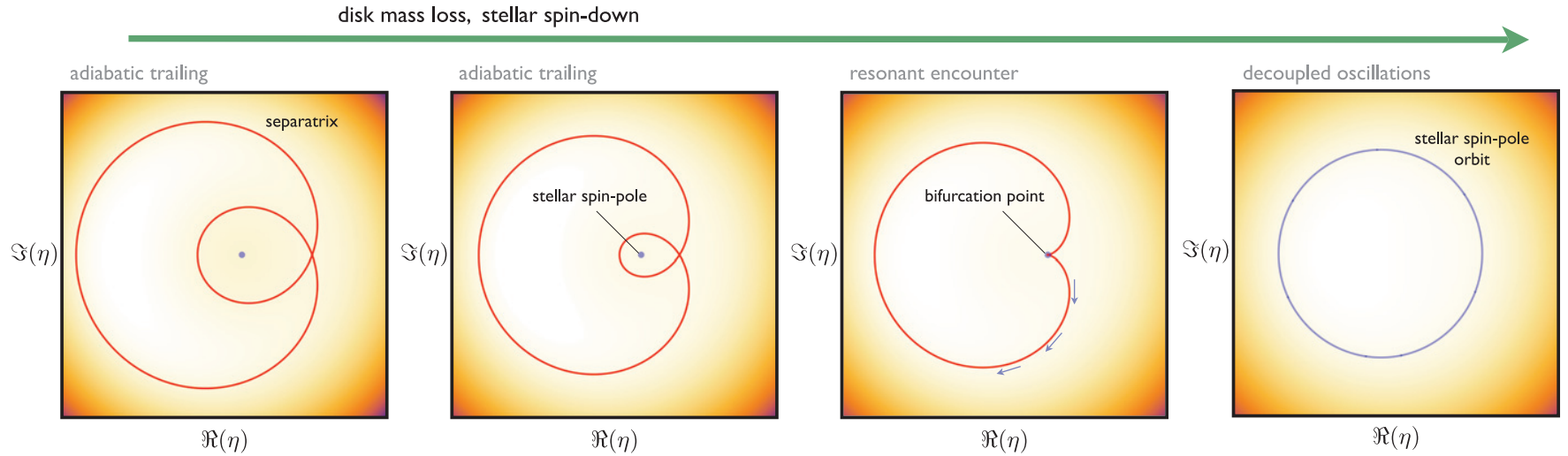

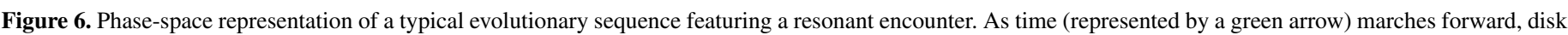

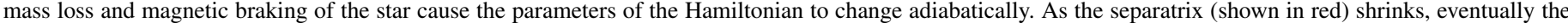

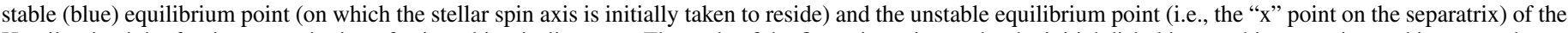

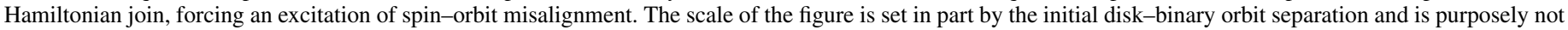
labeled. The background color is representative of level curves of the Hamiltonian.

(A color version of this figure is available in the online journal.)

As disk and stellar properties evolve with time, the characteristic regimes of the system will change. Thus, to obtain a quantitatively accurate solution for the stellar spin-axis dynamics, an explicitly time-dependent counterpart of the Hamiltonian (46) must be considered. Before doing so, however, we note that the overall behavior of the system can be understood qualitatively by examining the evolution of the above-mentioned timescales through a typical lifetime of the disk. Such a comparison is shown in Figure 5, where precession timescales corresponding to accreting and gravitationally contracting stars are shown in blue while those spun down by disk-locked magnetic torques are shown in red (see Figure 3). Additionally, the precession timescale for a star with a fixed rotational period of $P_{\star}=10$ days is shown by a purple dashed curve, and the accretion timescale is shown as a gray line. Meanwhile, the green shade represents a characteristic range of binary perturbation timescales of interest (see Batygin 2012 for an in-depth discussion).

Upon examining Figure 5, some conclusions about the likely nature of the solution can be drawn. First, the accretiondominated regime seems to be irrelevant for classical diskbearing $\mathrm{T}$ Tauri systems, although it is quite possible that this regime is pertinent during the embedded stage of a typical star's evolution. Second, the evolutionary difference between fast and slow rotators is striking: the precession timescale associated with stars that spin-up due to gravitational contraction and accretion remains approximately constant throughout the disk's lifetime and consistently straggles behind the average disk-torquing timescale. This means that fast rotators usually reside in the adiabatic trailing regime and misalignments can be expected to be rare. Meanwhile, the precession timescale of stars that successfully undergo magnetic braking increases by many orders of magnitude over a typical disk lifetime. Although such stars also start out adiabatically trailing the disks, the latter stages of their evolution are better characterized by the decoupled regime, where the disk-star inclination can reach substantial values. Note that the evolution of the precession timescale for a star rotating with a fixed period is qualitatively similar to that associated with a star that is magnetically spun down.

With the above treatment in mind, it is important to note that as the characteristic timescales evolve, there are (relatively short-lived) epochs when the precession and disk-torquing timescales are comparable. Until now, we have purposely avoided the discussion of such a (resonant) regime, because a commensurability between the frequencies $\mathcal{F}$ and $v$ leads to a singularity in the solution (48). To circumvent this problem, additional terms in the series expansion of the Hamiltonian must be retained. Following Lithwick \& Wu (2012), we incorporate an additional non-linear kinetic term, whose coefficient is coincidentally the same as that of the leading-order term, $f$, to the leading order in $\alpha$ (Murray \& Dermott 2000). Working through the transformations outlined above, the Hamiltonian takes the form

$$
\mathcal{H}=\mathcal{F}\left[\eta \eta^{*}-\frac{\left(\eta \eta^{*}\right)^{2}}{2}\right]-v\left[\eta \eta^{*}+\sqrt{Z_{0}^{\prime}}\left(\eta+\eta^{*}\right)\right]
$$

where the expansion in inclination is taken relative to the disk's frame.

The Hamiltonian (53) is a complex representation of the largely successful second fundamental model for resonance (Henrard \& Lamaitre 1983). Within the framework of this model and the closely related pendulum model, the theory of resonant encounters is well-established (for more in-depth treatments, see, e.g., Goldreich \& Peale 1966; Yoder 1973; Peale 1986; Henrard 1982), and qualitatively, the process boils down to the following picture. As system parameters change, the location of the homoclinic critical curve (the separatrix) evolves in phase space. When the actual trajectory of a system encounters the separatrix, the system experiences an impulsive change in action. A schematic phase-space representation of a typical evolutionary sequence where a resonant encounter occurs is shown in Figure 6.

Figure 7 shows a collection of solutions obtained by numerically integrating the equation of motion arising from the Hamiltonian (53), augmented with a dissipative term arising from accretion, as in Equation (47). The color scheme used here is the same as that in Figures 3 and 5. Specifically, solutions depicted with red curves are those where spin-down due to magnetic braking is evident and those depicted with blue curves correspond to rapidly rotating stars. The panels $\mathrm{A}, \mathrm{B}$, and C, depict solutions with the disk torquing timescale taken to be $\tau_{\text {torq }}=$ $0.3,1$, and $3 \mathrm{Myr}$, respectively. The inclination between the disk and the binary orbit is taken to be $i^{\prime}=30^{\circ}$ across the panels. 


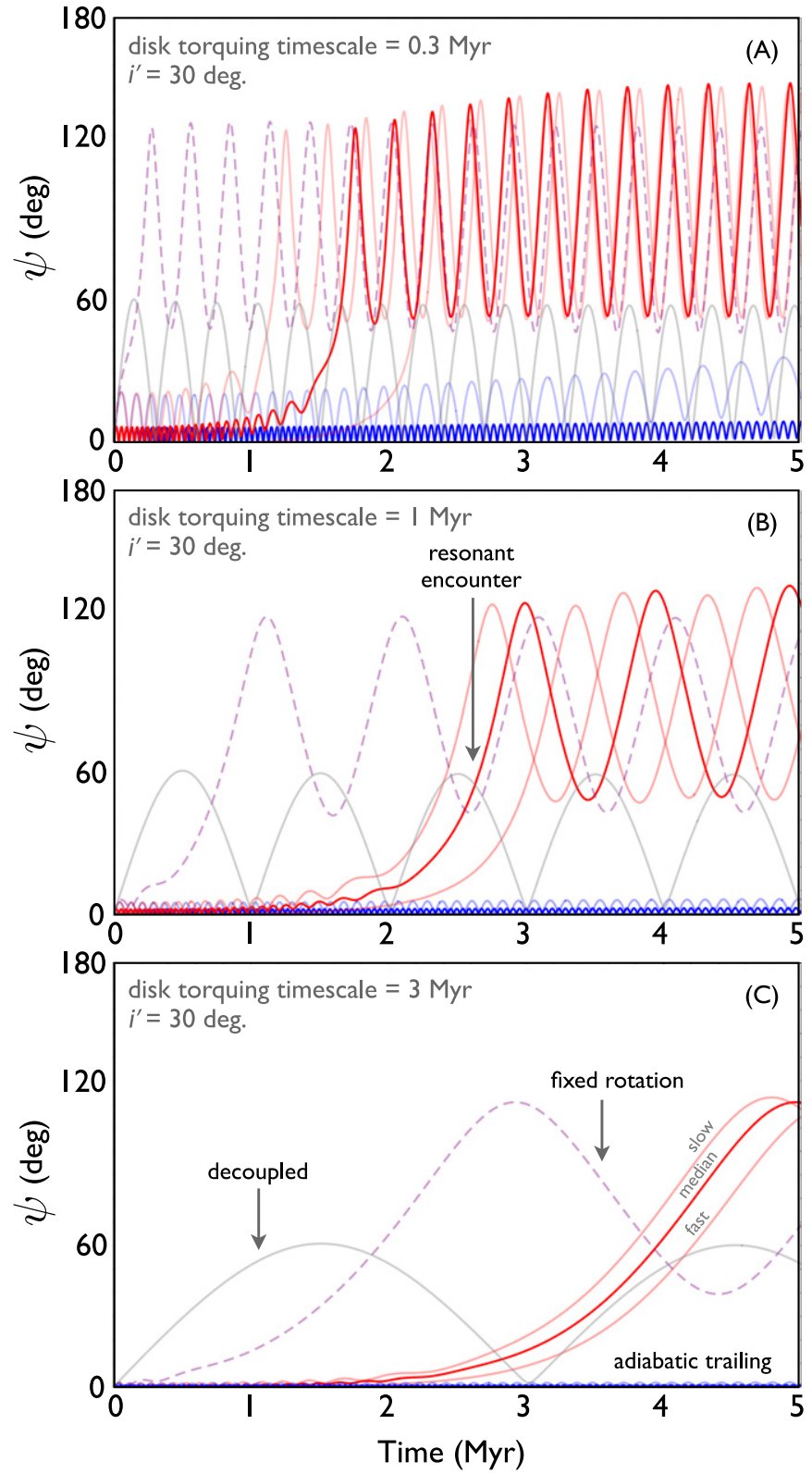

Figure 7. Misalignment angle as a function of time computed within the framework of the nonlinear Hamiltonian from Equation (53), augmented with a simple prescription for accretion-driven re-alignment of the star. The utilized color scheme is largely the same as that used in Figures 3 and 5. For completeness, a solution with $\mathcal{F}=0$ is also shown as a gray line. The three panels of the figure depict solutions with different disk-torquing timescales, as labeled. The initial disk-binary orbit misalignment is taken to be $i^{\prime}=30$ degrees across the panels. Note that because the solution is obtained from a nonlinear Hamiltonian, the result will not necessarily scale with the disk-binary inclination, as in the case of the linear solution of Equation (48).

(A color version of this figure is available in the online journal.)

Evidently, two regimes characterize the solutions, depending on the rotational evolution of the star. As expected from Figure 5, the spin axes of stars that are spun up due to gravitational contraction and accretion adiabatically trail the disk, leading to consistently low misalignments. Stars that are gradually spun down by magnetic disk-star coupling encounter a resonance between the spin-axis precession frequency and the disk torquing frequency. As discussed above, this results in an impulsive excitation of mutual inclination that is followed by oscillations characteristic of the decoupled regime. Similar behavior can be seen for the case where the stellar rotation period is held fixed (shown with a dashed purple line). For completeness, solutions corresponding to a perfectly spherical star such as those considered by Batygin (2012), which reside in the decoupled regime by definition, are also shown with gray curves.

Cumulatively, our calculations show that substantial star-disk inclinations naturally arise for a wide range of binary orbit configurations, a proxy for which is the disk-torquing timescale. However, a controlling feature that determines the feasibility of misalignment excitation is stellar rotational evolution. For disk torquing timescales on the order of $\sim \mathrm{Myr}$ or longer, fast rotators gravitationally lock to the disk inhibiting the onset of misalignment, while median and slow rotators tilt as a result of an encounter with a secular stellar precession-disk torquing resonance. Although the latter holds true for faster disk-torquing timescales, as elucidated by panel A of Figure 7, free oscillations of the misalignment angle can be substantial even for rapid rotators. Accordingly, the inherent breadth of the distribution of rotation rates of young stars appears to be an essential feature, required for the reproduction of the observed wide-ranging distribution of Hot Jupiter spin-orbit misalignments.

\section{CONCLUSION AND DISCUSSION}

In this paper, we have characterized long-term disk-star interactions with an eye toward consolidating the rotational evolution of young stellar objects and the generation of spin-orbit misalignments into a unified framework. In this initial effort, we have placed significant emphasis on the analytic treatment of physics, yielding an approximate yet more or less transparent model that captures the basic ingredients of the problem at hand.

We began by considering the evolution of stellar rotation rates, accounting for gravitational contraction, accretion, and magnetic torques. Our calculations show that depending on the relaxation or imposition of the disk-locking assumption, stars end up with rotation rates that fall in the observed range of $P=1-10$ days (Herbst et al. 2002; Littlefair et al. 2010; Affer et al. 2013) after several Myr. Stellar spin-down appears to be dominated by magnetic torques, while gravitational contraction dominates stellar spin-up. A careful account for the geometry of the magnetic field suggests that accretion is a sub-dominant process. The parity of gravitational contraction and magnetic braking yields considerable diversity in spin rates within the observed range, where the value for a particular system depends on the exact choice of parameters. The heterogeneity inherent to rotational evolution is further enhanced by the corrections to the spin-up torques introduced by accretion and its sensitive dependence on the dipole/octupole morphology of the magnetic field.

Although we have not considered this complication in our calculations, it is noteworthy that the dipole/octupole dichotomy is also likely to add diversity to the evaluation of magnetic torques. As an example, consider a pair of stars with the same surface field strengths but different field structures. Because an octupole field decays more rapidly with distance, we can expect a weaker magnetic field at the disk coupling point, implying a smaller spin-down torque compared to the dipole-dominated counterpart (Livio \& Pringle 1992; Matt \& Pudritz 2005). Further complexity is introduced by the fact that the magnetic poles can be tilted with respect to the rotational poles, and the dipole/ octupole orientations can exhibit large inclinations with respect to each other. All of these considerations may give rise to 
substantial quantitative changes of the solutions shown in Figure 3 as well as greater difficulty in trying to ascertain which stars end up with which rotational periods.

In the latter half of the paper, we considered the spin-axis dynamics of rotationally evolving, disk-bearing stars subject to external torques arising from binary companions. Using orbit-averaged perturbation theory, we demonstrated that large misalignments between disk orbital angular momentum vectors and stellar spin axes are preferentially excited in systems where rotational evolution features a significant spin-down of the host star. Specifically, in nearly all of the solutions we obtained (with the exception of those characterized by rapid disk-torquing timescales), gravitational coupling between the disk and the stellar gravitational quadrupole moment (which arises from rotational deformation) played a dominant role in quenching spin-orbit misalignment at early epochs. However, as the disk mass declined, allowing gravitational coupling to subside, stars whose rotation rates decreased (or were held constant) experienced an impetuous excitation of spin-orbit misalignment as a result of an encounter with a disk-star precession resonance. Such a resonance was never encountered for stars whose rotation rates monotonically increased throughout the disk's lifetime. In either case, star-disk realignment due to accretion was consistently negligible.

It is important to understand that the manner in which the spin-orbit misalignment originates within the calculations presented here is qualitatively different from that envisioned by Batygin (2012). That is, the decoupled regime characteristic of the solutions presented by Batygin (2012) is only observed after the initial orbital obliquity is seeded. Indeed, the realization of an impulsive acquisition of spin-orbit misalignment due to the crossing of a secular resonance between the disk-torquing frequency and the stellar precession frequency is the key result of this paper.

The theory for the origin of hot Jupiter spin-orbit misalignments presented here can be confirmed or refuted observationally. The most direct avenue for doing so is a survey aimed at detection and characterization of close-in giant planets around $\mathrm{T}$ Tauri stars. If disk-driven migration (as opposed to dynamical $\mathrm{N}$-body interactions) is responsible for the production of hot Jupiters, the fraction of hot Jupiter-bearing weak-lined T Tauri stars should be commensurate with (or perhaps greater than) that of field stars, i.e., $\gtrsim 1 \%$ (Wright et al. 2011). Our model would thus predict that among such a sample, planets with high obliquities would preferentially reside in multiple stellar systems and orbit slowly rotating stars. To date, with the exception of the recent work of Barnes et al. (2013), such observations remain scarce.

Another test of our model for spin-orbit misalignment may arise from measurements of the Rossiter-McLaughlin effect in systems of multiple low-mass, multi-transiting planets, many of which have now been discovered by the Kepler mission (Batalha et al. 2013). Because the calculations presented here do not consider planetary mass as an inherent parameter, the mechanism for the acquisition of orbital obliquity should be oblivious to the specific nature of the planetary system at hand. In fact, for our purposes, even the question of distant (beyond the ice line) formation followed by extensive disk-driven migration (Kley \& Nelson 2012 and the references therein) versus in situ formation (Hansen \& Murray 2012; Chiang \& Laughlin 2013) should not play an appreciable role. Studies of this sort are already underway (Albrecht et al. 2013) although the number of analyzed systems still remains small. Finally, considerable insight into the importance of the mechanism outlined in this paper may be gleamed from a comparison of the theoretically obtained distribution of spin-orbit misalignments and its observed counterpart. Such an analysis will be published as a follow-up study (Crida \& Batygin 2013).

Independent of the aforementioned tests, there exists an additional observational fact, relevant to our model. The current aggregate of data associated with the Rossiter-McLaughlin effect suggests that the existence of this misalignment depends on stellar mass or photospheric temperature $T_{\text {eff }}$. More specifically, the misalignments are essentially null for values of $T_{\text {eff }}$ below a critical value of $T_{C} \approx 6250 \mathrm{~K}$ and can be arbitrarily large (that is, ranging from prograde and aligned, to retrograde and anti-aligned) for hotter stars. The theoretical basis for this dichotomy remains controversial. Winn et al. (2010) and subsequently, Albrecht et al. (2012) have argued that the polarity of the observations reflects the changes in efficiency of tidal dissipation with stellar type (Lai et al. 2011; Lai 2012). Specifically, these authors envisioned a scenario where all hot Jupiters arrive onto their orbits with high inclinations, that are subsequently erased in low-mass stars due to efficient tidal dissipation in the convective parts of the envelope. However, this story was recently challenged by Rogers \& Lin (2013), who argued that tidal dissipation preferentially leads to prograde aligned, retrograde aligned, or orthogonal spin-orbit angles, in some contradiction with the observed distribution.

In principle, the mechanism discussed here is consistent with the narrative of Winn et al. (2010). That is, one can envision an initially isotropic distribution of orbital obliquities, that is further shaped by tidal dissipation within the framework of our mechanism. However, it is likely that an alternative scenario may also be conjured up. Specifically, based on the interdependence of rotational evolution and disk-star interactions, we may hypothesize that the observed mass-dependence inherent to rotational evolution (Bouvier 2013) may be responsible for the observed dependence of spin-orbit misalignment on $T_{\text {eff. }}$ A quantitative examination of this speculative idea would require enhanced understanding of PMS star-disk angular momentum transfer.

In light of the arguments presented above, it is clear that in addition to an enhanced aggregate of observations, a meaningful comparison between the data and the model will require further theoretical developments. Some enhancements to the treatment at hand are straightforward. For example, our polytropic structure models can be replaced with state-of-the-art stellar evolution calculations (Paxton et al. 2013). Moreover, our perturbative treatment of gravitational star-disk interactions (that assumes small inclinations) can be easily cast into a more precise form (Colombo 1966; Henrard 1991). On the other hand, the entirety of the disk-locking process is complicated and imperfectly understood (see Barnes et al. 2001; Matt \& Pudritz 2004; Long et al. 2005; Zanni \& Ferreira 2009 and references therein). Consequently, the construction of a significantly more detailed iteration of our model may be constrained by an incomplete understanding of magnetic disk-star coupling. In any case, however, the available observational data suggests that our treatment of rotational evolution (although approximate) is plausible and may be sufficient for the purposes at hand.

In conclusion, the model developed in this work opens up a previously unexplored avenue toward understanding star-disk evolution and its consequences in a unified manner. Although a great deal of additional effort, both observational and theoretical, is required to elucidate all of the physics involved, the 
novel mechanism presented here provides a promising way to understand a number of observed features of star-disk-planet systems.

We thank the referee, Scott Gregory, for providing an exceptionally helpful and thorough report that resulted in a substantial improvement of the manuscript. K.B. acknowledges generous support from the ITC Prize Postdoctoral Fellowship at the Institute for Theory and Computation, Harvard-Smithsonian Center for Astrophysics. F.C.A. acknowledges support from NASA Origins grant NNX11AK87G.

\section{REFERENCES}

Adams, F. C. 2010, ARA\&A, 48, 47

Adams, F. C. 2011, ApJ, 730, 27

Adams, F. C., \& Gregory, S. G. 2012, ApJ, 744, 55

Affer, L., Micela, G., Favata, F., Flaccomio, E., \& Bouvier, J. 2013, MNRAS, 430,1433

Albrecht, S., Winn, J. N., Johnson, J. A., et al. 2012, ApJ, 757, 18

Albrecht, S., Winn, J. N., Marcy, G. W., et al. 2013, ApJ, 771, 11

Anderson, K. R., Adams, F. C., \& Calvet, N. 2013, ApJ, 774, 9

Armitage, P. J., \& Clarke, C. J. 1996, MNRAS, 280, 458

Barnes, J. W., van Eyken, J. C., Jackson, B. K., Ciardi, D. R., \& Fortney, J. J. 2013, ApJ, 774, 53

Barnes, S., Sofia, S., \& Pinsonneault, M. 2001, ApJ, 548, 1071

Batalha, N. M., Rowe, J. F., Bryson, S. T., et al. 2013, ApJS, 204, 24

Bate, M. R., Lodato, G., \& Pringle, J. E. 2010, MNRAS, 410, 1505

Batygin, K. 2012, Natur, 491, 418

Batygin, K., Morbidelli, A., \& Tsiganis, K. 2011, A\&A, 533, A7

Beaugé, C., \& Nesvorný, D. 2012, ApJ, 751, 119

Belyaev, M. A., Rafikov, R. R., \& Stone, J. M. 2013, ApJ, 770, 68

Binney, J., \& Tremaine, S. 1987, in Galactic Dynamics, (Princeton, NJ: Princeton Univ. Press),

Bouvier, J. 2013, arXiv:1307.2891

Caratti o Garatti, A., Garcia Lopez, R., Scholz, A., et al. 2011, A\&A, 526, 1

Chaboyer, B., Demarque, P., \& Pinsonneault, M. H. 1995, ApJ, 441, 876

Chandrasekhar, S. 1939, in An Introduction to the Study of Stellar Structure, (Chicago, IL: Univ. Chicago Press)

Charbonneau, D., Brown, T. M., Latham, D. W., \& Mayor, M. 2000, ApJL, 529, L45

Chiang, E., \& Laughlin, G. 2013, MNRAS, 431, 3444

Colombo, G. 1966, AJ, 71, 891

Crida, A., \& Batygin, K. 2013, AAS/Division for Planetary Sciences Meeting Abstracts 45, 300.02

Donati, J.-F., Jardine, M. M., Gregory, S. G., et al. 2007, MNRAS, 380, 1297

Donati, J.-F., Jardine, M. M., Gregory, S. G., et al. 2008, MNRAS, 386, 1234

Donati, J., Skelly, M. B., Bouvier, J., et al. 2010, MNRAS, 409, 1347

Donati, J.-F., Bouvier, J., Walter, F. M., et al. 2011, MNRAS, 412, 2454

Donati, J.-F., Gregory, S. G., Alencar, S. H. P., et al. 2012, MNRAS, 425, 2948

Evans, N. J., II, Dunham, M. M., Jørgensen, J. K., et al. 2009, ApJS, 181, 321

Fabrycky, D., \& Tremaine, S. 2007, ApJ, 669, 1298

Fischer, D. A., \& Valenti, J. 2005, ApJ, 622, 1102

Ford, E. B., \& Rasio, F. A. 2008, ApJ, 686, 621

Gallet, F., \& Bouvier, J. 2013, arXiv:1306.2130

Ghez, A. M., Neugebauer, G., \& Matthews, K. 1993, AJ, 106, 2005

Ghosh, P., \& Lamb, F. K. 1978, ApJL, 223, L83

Goldreich, P., \& Peale, S. J. 1966, AJ, 71, 425

Goldreich, P., \& Tremaine, S. 1980, ApJ, 241, 425

Gregory, S. G., \& Donati, J.-F. 2011, AN, 332, 1027

Gregory, S. G., Donati, J.-F., Morin, J., et al. 2012, ApJ, 755, 97

Gregory, S. G., Jardine, M., Gray, C. G., \& Donati, J.-F. 2010, RPPh, 73, 126901

Hansen, B. M. S., \& Murray, N. 2012, ApJ, 751, 158

Hansen, C. J., \& Kawaler, S. D. 1994, in Stellar Interiors: Physical Principles, Structure, and Evolution, (New York: Springer)

Hartmann, L. 2008, PhyS, 130, 014012

Hartmann, L., Calvet, N., Gullbring, E., \& D'Alessio, P. 1998, ApJ, 495, 385

Hartmann, L., D’Alessio, P., Calvet, N., \& Muzerolle, J. 2006, ApJ, 648, 484

Henrard, J. 1982, CeMec, 27, 3

Henrard, J. 1991, in Predictability, Stability, and Chaos in N-Body Dynamical Systems, ed. A. E. Roy (New York, NY: Plenum Press), 193

Henrard, J., \& Lamaitre, A. 1983, CeMec, 30, 197
Herbst, W., Eislöofffe, J., Mund, R., \& Scholz, A. 2007, in Protostars and Planets V, ed. B. Reipurth, D. Jewitt, \& K. Keil (Tucson, AZ: Univ. Arizona Press), 297

Herbst, W., Hamilton, C. M., Vrba, F. J., et al. 2002, PASP, 114, 1167

Herczeg, G., \& Hillenbrand, L. A. 2008, ApJ, 681, 594

Hernández, J., Hartmann, L., Megeath, T., et al. 2007, ApJ, 662, 1067

Hillenbrand, L. A. 2008, PhyS, 130, 014024

Hillenbrand, L. A., Strom, S. E., Vrba, F. J., \& Keene, J. 1992, ApJ, 397, 613

Jackson, J. D. 1998, Classical Electrodynamics, ed. J. D. Jackson (3rd ed.; New York: Wiley-VCH), 832

Johns-Krull, C. M. 2007, ApJ, 664, 975

Kawaler, S. D. 1988, ApJ, 333, 236

Kley, W., \& Nelson, R. P. 2012, ARA\&A, 50, 211

Königl, A. 1991, ApJL, 370, L39

Kraus, A. L., Ireland, M. J., Martinache, F., \& Hillenbrand, L. A. 2011, ApJ, 731, 8

Lada, C. J., \& Lada, E. A. 2003, ARA\&A, 41, 57

Lai, D. 2012, MNRAS, 423, 486

Lai, D., Foucart, F., \& Lin, D. N. C. 2011, MNRAS, 412, 2790

Lamm, M. H., Mundt, R., Bailer-Jones, C. A. L., \& Herbst, W. 2005, A\&A, 430, 1005

Laughlin, G., Bodenheimer, P., \& Adams, F. C. 2004, ApJL, 612, L73

Lee, M. H., \& Peale, S. J. 2002, ApJ, 567, 596

Lin, D. N. C., Bodenheimer, P., \& Richardson, D. C. 1996, Natur, 380, 606

Lithwick, Y., \& Wu, Y. 2012, ApJL, 756, L11

Littlefair, S. P., Naylor, T., Mayne, N. J., Saunders, E. S., \& Jeffries, R. D. 2010, MNRAS, 403, 545

Livio, M., \& Pringle, J. E. 1992, MNRAS, 259, 23P

Long, M., Romanova, M. M., \& Lovelace, R. V. E. 2005, ApJ, 634, 1214

Marcy, G., \& Butler, P. R. 1996, ApJL, 464, L147

Marks, M., \& Kroupa, P. 2012, A\&A, 543, 8

Matt, S., \& Pudritz, R. E. 2004, ApJ, 607, 43

Matt, S., \& Pudritz, R. E. 2005, MNRAS, 356, 167

Matt, S., \& Pudritz, R. E. 2008, ApJ, 678, 1109

Matt, S. P., Pinzón, G., Greene, T. P., \& Pudritz, R. E. 2012, ApJ, 745, 101

Mayor, M., \& Queloz, D. 1995, Natur, 378, 355

McLaughlin, D. B. 1924, ApJ, 60, 22

Moffatt, H. K. 1978, Magnetic Field Generation in Electrically Conducting Fluids (Cambridge: Cambridge Univ. Press)

Mohanty, S., \& Shu, F. H. 2008, ApJ, 687, 1323

Morbidelli, A. 2002, Modern Celestial Mechanics: Aspects of Solar System dynamics (London: Taylor and Francis)

Morbidelli, A., \& Crida, A. 2007, Icar, 191, 158

Morbidelli, A., Tsiganis, K., Batygin, K., Crida, A., \& Gomes, R. 2012, Icar, 219,737

Murray, C. D., \& Dermott, S. F. 2000, Solar System Dynamics (Cambridge: Cambridge Univ. Press)

Nagasawa, M., Ida, S., \& Bessho, T. 2008, ApJ, 678, 498

Naoz, S., Farr, W. M., Lithwick, Y., Rasio, F. A., \& Teyssandier, J. 2011, Natur, 473, 187

Narayan, R., Goldreich, P., \& Goodman, J. 1987, MNRAS, 228, 1

Paxton, B., Cantiello, M., Arras, P., et al. 2013, arXiv:1301.0319

Peale, S. J. 1986, in Satellites, ed. J. A. Burns \& M. S. Matthews (Tucson, AZ: Univ. Arizona Press), 159

Phillips, A. C. 1994, The Physics of Stars (Chichester: Wiley)

Pollack, J. B., Hubickyj, O., Bodenheimer, P., et al. 1996, Icar, 124, 62

Radoski, H. R. 1967, JGR, 72, 418

Rogers, T. M., \& Lin, D. N. C. 2013, ApJ, 769, 10

Rogers, T. M., Lin, D. N. C., \& Lau, H. H. B. 2012, ApJL, 758, L6

Romanova, M. M., Ustyugova, G. V., Koldoba, A. V., \& Lovelace, R. V. E. 2002, ApJ, 578, 420

Romanova, M. M., Ustyugova, G. V., Koldoba, A. V., Wick, J. V., \& Lovelace,

R. V. E. 2003, ApJ, 595, 1009

Rossiter, R. A. 1924, ApJ, 60, 15

Sekiya, M., Nakazawa, K., \& Hayashi, C. 1980, PThPh, 64, 1968

Shakura, N. I., \& Sunyaev, R. A. 1973, A\&A, 24, 337

Shu, F., Najita, J., Ostriker, E., et al. 1994, ApJ, 429, 781

Siess, L., Dufour, E., \& Forestini, M. 2000, A\&A, 358, 593

Stahler, S. W., \& Palla, F. 2005, The Formation of Stars (Weinheim: Wiley-VCH)

Stanley, S., \& Bloxham, J. 2004, Natur, 428, 151

Struve, O. 1952, Obs, 72, 199

Uzdensky, D. A., Königl, A., \& Litwin, C. 2002, ApJ, 565, 1191

Van Laerhoven, C., \& Greenberg, R. 2012, CeMDA, 113, 215

Williams, J. P., \& Cieza, L. A. 2011, ARA\&A, 49, 67

Winn, J. N., Albrecht, S., Johnson, J. A., et al. 2011a, ApJ, 741, 1 
Winn, J. N., Fabrycky, D., Albrecht, S., \& Johnson, J. A. 2010, ApJL, $718, \mathrm{~L} 145$

Winn, J. N., Howard, A. W., Johnson, J. A., et al. 2011b, AJ, 141, 63

Winn, J. N., Johnson, J. A., Peek, K. M. G., et al. 2007, ApJL, 665, L167

Wolszczan, A., \& Frail, D. A. 1992, Natur, 355, 145
Wright, J. T., Fakhouri, O., Marcy, G. W., et al. 2011, PASP, 123, 412

Wu, Y., \& Lithwick, Y. 2011, ApJ, 735, 109

Wu, Y., \& Murray, N. 2001, ApJ, 589, 605

Yoder, C. F. 1973, PhD thesis, U. C. Santa Barbara

Zanni, C., \& Ferreira, J. 2009, A\&A, 508, 1117 